\title{
The effect of surface contamination on the drift instability of standing Faraday waves
}

\author{
By ELENA MARTÍN AND JOSÉ M. VEGA \\ E. T. S. Ingenicros Industriales, Universidad de Vigo, Campus Lagoas, Marcosende, 9, \\ 36200-Vigo, Pontevedra, Spain \\ E. T. S. Ingenieros Acronáuticos, Universidad Politécnica de Madrid, Plaza Cardenal \\ Cisneros, 3, 28040-Madrid, Spain
}

We consider the effect of surface contamination, modelled by Marangoni clasticity with insoluble surfactant and surface viscosity, in promoting drift instabilities in spatially uniform standing Faraday waves. It is seen that contamination enhances drift instabilitics that lead to various stcadily propagating and (both standing and propagating) oscillatory patterns. In particular, steadily propagating waves appear to be quite robust, as a seminal experiment by Douady, Fauve \& Thual (1989).

\section{Introduction and formulation}

Surface contamination is likely to be present in water unless care is taken in the experimental set-up. This effect is usually modelled by phenomenological formulac (Dorrestcin 1951; Levich 1962; Miles 1967; Henderson 1998) based on Marangoni elasticity with insoluble surfactant and has been seen to dramatically increase the damping rate of gravity capillary waves, as first shown by Dorrestein (1951); an analysis intending to explain some surface wave damping measurements by Henderson \& Miles (1994) was made by Nicolás \& Vega (2000), who also anticipated that surface contamination would enhance the generation of the streaming flow produced by the surface wave. The streaming flow, in turn, affects the dynamics of the primary surface waves in Faraday systems (Vega, Knobloch \& Martel 2001; IIiguera, Vega \& Knobloch 2002; Lapuerta, Martel \& Vega 2002) and, in particular, plays a fundamental role in promoting drift instabilitics of standing Faraday waves (Martín, Martel \& Vega 2002, hereinafter referred to as MMV). The latter instabilities were experimentally observed by Douady, Fauve \& Thual (1989) in an annular container. I'he liquid was tap water, which is probably contaminated, while the analysis by MMV assumed a clean free surface. The object of this paper is to extend the analysis in MMV to the contaminated case. 'lhis extension is necessary because drift instabilities were quite robust in the above mentioned experiment (Fauve, personal communication 2004), while they were sensitive to various parameters such as the aspect ratio in the thcory for a clcan frec surfacc.

As in MMV, and with the same notation, we consider a horizontal two-dimensional liquid layer supported by a vertically vibrating plate (figure 1), and use the container's depth $h$ and the gravitational time $\sqrt{h / g}$ for non-dimensionalization. The governing equations are

$$
\begin{gathered}
u_{x}+v_{y}=0, \\
u_{t}+v\left(u_{y}-v_{x}\right)=-q_{x}+C\left(u_{x x}+u_{y y}\right),
\end{gathered}
$$




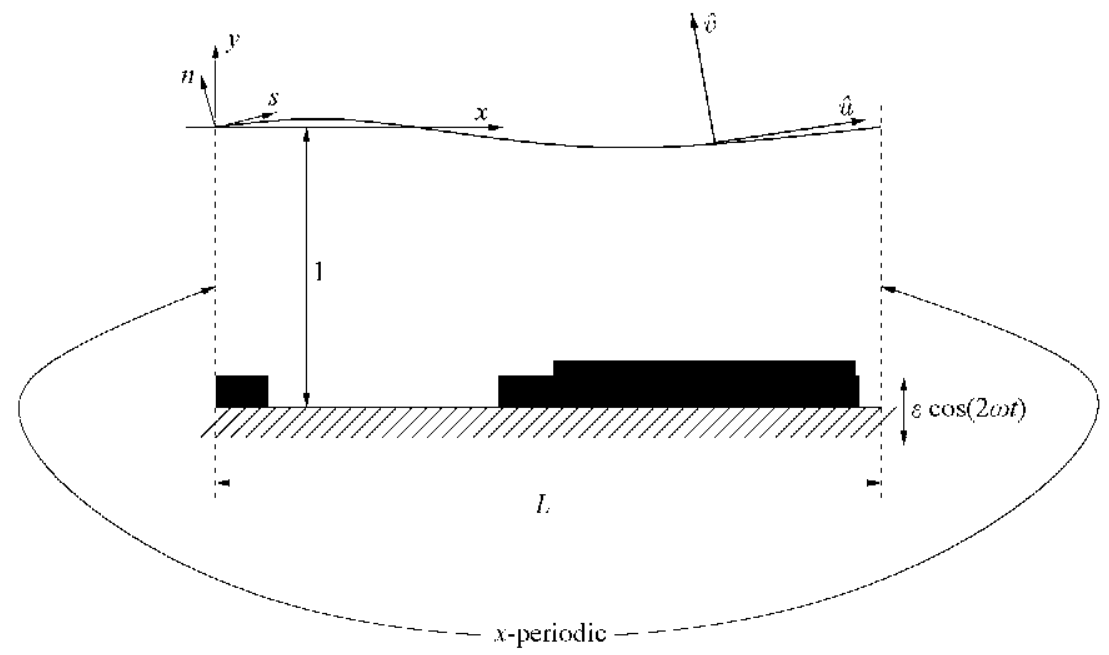

FIGLRE 1. Sketch of the fluid domain.

$$
\begin{gathered}
v_{t}-u\left(u_{y}-v_{x}\right)=-q_{y}+C\left(v_{x x}+v_{y y}\right), \\
u=v=0 \text { at } \quad y=-1, \\
v=f_{t}+u f_{x}, \quad c^{1 / 2}\left(\hat{u}_{n}+\hat{v}_{s}+\kappa \hat{u}\right)=-\gamma \zeta_{s}+\delta \hat{u}_{s s}, \\
q-\frac{1}{2}\left(u^{2}+v^{2}\right)+4 \omega^{2} \varepsilon f \cos (2 \omega t)-f+T \kappa=2 C \hat{v}_{n} \quad \text { at } y=f, \\
u, v, q \text { and } f \text { are } L \text {-periodic in } x,
\end{gathered}
$$

where

$$
s=\int_{0}^{x} \sqrt{1+f_{x}^{2}} \mathrm{~d} x, \quad \kappa=\frac{f_{x x}}{\left(1+f_{x}^{2}\right)^{3 / 2}},
$$

are an arc length parameter and the curvature of the free surface (defined as $y=f$ ), respectively, and $n$ is a coordinate along the upward unit normal to the frec surface; note that

$$
\frac{\partial}{\partial s}=\frac{1}{\sqrt{1+\int_{x}^{2}}}\left(\frac{\partial}{\partial x}+f_{x} \frac{\partial}{\partial y}\right), \quad \frac{\partial}{\partial n}=\frac{1}{\sqrt{1+\int_{x}^{2}}}\left(\frac{\partial}{\partial y}-f_{x} \frac{\partial}{\partial x}\right) .
$$

$\hat{u}$ and $\hat{v}$ are the tangential and normal velocity components, which are related to the horizontal and vertical components by

$$
\hat{u}=\frac{u+f_{x} v}{\sqrt{1+f_{x}^{2}}}, \quad \hat{v}=\frac{v-f_{x} u}{\sqrt{1+f_{x}^{2}}} .
$$

The only difference with MMV is the boundary condition (1.5b), whose right-hand side was zero and now accounts for the presence of contaminating surfactants, modelled in the simplest way: the resulting tangential stress includes Marangoni clasticity effects and surface viscosity. The latter is based on the Boussinesq-Scriven surface model. Scriven (1960) generalized the mathematical description of the Boussinesq (1913) treatment for a time-dependent interface for which, in addition to its dependence on the surface tension gradients, the interfacial stress is a (linear) function of two other intrinsic propertics of the interface, namcly the surface shear viscosity $\mu_{1}^{s}$ and the surface dilatational viscosity $\mu_{2}^{S}$, both assumed constants here. The (two-dimensional) surface stress is written as

$$
\boldsymbol{\tau}=\nabla_{S} T^{*}+\left(\mu_{2}^{S}-\mu_{1}^{S}\right) \nabla_{S}\left(\nabla_{S} \cdot \boldsymbol{v}^{S}\right)+\mu_{1}^{S} \nabla_{S} \cdot\left[\nabla_{S} \boldsymbol{v}^{S}+\left(\nabla_{S} \boldsymbol{v}^{S}\right)^{\top}\right],
$$


where $\nabla_{S}$ is the (two-dimensional) surface gradient operator, $v^{S}$ is the (twodimensional) surface velocity vector, and $T$ denotes the transpose. The variation of the surface tension with the surfactant concentration $\zeta^{*}$ is approximated by a linear law of the form $T^{*}\left(\zeta^{*}\right)=T_{0}^{*}+\left(\mathrm{d} T^{*} / \mathrm{d} \zeta_{0}^{*}\right)\left(\zeta^{*}-\zeta_{0}^{*}\right)$, where the derivative is calculated at the equilibrium value of $\zeta^{*}$, denoted as $\zeta_{0}^{*}$. The boundary condition (1.5b) results from equating the surface stress to the viscous shear stress from the bulk at the free surface, and non-dimensionalizing. It follows that the Marangoni elasticity and the non-dimensional surface viscosity are given by $\gamma=\zeta_{0}^{*}\left(\mathrm{~d} T^{*} / \mathrm{d} \zeta_{0}^{*}\right) C^{1 / 2} /(\mu \sqrt{g h})$ and $\delta=\left(\mu_{1}^{S}+\mu_{2}^{S}\right) \sqrt{C} /(\mu h)$. The non-dimensional surfactant concentration $\zeta=\left(\zeta^{*}-\zeta_{0}^{*}\right) / \zeta_{0}^{*}$ is given by the conservation equation

$$
\zeta_{t}+\lceil(1+\zeta) u]_{s}=0 \quad \text { in } 0<s<s_{L}, \quad \zeta\left(s+s_{L}, t\right)=\zeta(s, t)
$$

IIere, $s_{L}$ is the length of the free surface in one period and we are neglecting both cubic terms and surface diffusion of the surfactant. Note that surface tension variation duc to the presence of surfactant is of the order of $\gamma \zeta_{s} \sqrt{C}$, which is assumed small compared with $T$ (with $C$ and $T$ defined below); thus this variation is ignored in the normal stress balance $(1.5 \mathrm{c})$.

The problem depends on the following non-dimensional parameters: the forcing frequency $2 \omega=2 \omega^{*} \sqrt{h / g}$ and amplitude $\varepsilon=\varepsilon^{*} / h$, the ratio of viscous to gravitational cffects $C=\mu /\left(\rho \sqrt{g h^{3}}\right)$, the Bond number $T^{-1}=\rho g h^{2} / T_{0}^{*}$, and the horizontal aspect ratio $L=L^{*} / h$, where $L^{*}$ is the horizontal length.

We shall consider small nearly-resonant solutions at small viscosity and conveniently rescaled Marangoni elasticity, i.e.

$$
|u|+|v|+|q|+|f|+|\zeta| \ll 1, \quad \varepsilon \ll 1, \quad\left|\omega-\omega_{0}\right| \ll 1, \quad C \ll 1, \quad \gamma \sim 1 .
$$

I Iere, $\omega_{0}$ is a natural frequency in the inviscid limit, and the assumption that $C \ll 1$ is reasonable for fluids with a sufficiently small viscosity in not too thin layers $\left(C \sim 10^{-4}\right.$ for water in $1 \mathrm{~cm}$ decp containers, as in the experiment by Douady et al. 1989). The latter assumption $(\gamma \sim 1)$ is made for the Marangoni elasticity to have a significant effect both in the damping ratio of the surface waves and in the streaming flow. Ihis assumption gives the best comparison with the damping ratio measurements by Henderson \& Miles (1994), as explained by Nicolas \& Vega (2000), and it is satisfied for a $1 \mathrm{~cm}$ decp layer and surfactants such as those in the experiment by Henderson (1998), where $\zeta_{0}^{*} \mathrm{~d} T^{*} / \mathrm{d} \zeta_{0}^{*}=14,21$, and $7 \mathrm{dyn} \mathrm{cm}^{-1}$ for lecithin, oleyl alcohol and dyolein, respectively.

We do not have any a priori knowledge concerning the valuc of surface viscosity in contaminated water, except the guess that $\delta$ should be somewhat small compared to $\gamma$. The results in this paper apply also to (i) the effect of controlled surfactants when using other liquids (I Ienderson 1998) and (ii) the case when a film of an immiscible liquid is deposited above the free surface (Jenkins \& Dysthe 1997); the effective surface viscosity in this case is $\mu_{s}=\mu_{V} d^{*}$, where $\mu_{V}$ is the volumetric viscosity of the liquid, and $d^{*}$ is the film thickness. In cases (i) and (ii), surface viscosity can exhibit much higher values (Jenkins \& Dysthe 1997; Hirsa, López \& Miraghaic 2002) than in contaminated water. For instance, in case (ii), we note that Newtonian liquids (e.g. Dow 200 Silicone oil) in the film can exhibit a viscosity as high as $1000 \mathrm{St}$, namely $10^{5}$ limes the viscosity of water, which yiclds quite large effective surface viscosity for moderately small film thickness. Thus, in potentially interesting cases, $\delta$ varies in a wide range, from quite small to quite large values. 
For convenience, we note that the overall surfactant concentration and the overall shear stress on the free surface are conserved, i.c.

$$
\int_{0}^{s_{L}}\left[\hat{u}_{n}(s, t)+\kappa(s, t) \hat{u}(s, t)\right] \mathrm{d} s=0, \quad \int_{0}^{s_{L}} \zeta(s, t) \mathrm{d} s=0,
$$

as obtained integrating $(1.5 b)$ and $(1.10 a)$ in one period and applying the periodicity conditions. I'he former condition is a consequence of the fact that the massless free surface cannot absorb any overall shear stress from the fluid. This condition also applies when the two-dimensional layer considered here models an annular container whose non-dimensional width $\ell$ is large (but small compared to the container radius, to avoid curvature effects) provided that the surface viscosity is conveniently small. In fact, since $\hat{u}=0$ at the lateral walls, the two-dimensional version of $(1.5 b)$ in the annular container readily yields the following estimate

$$
\sqrt{C} \ell\left|\int_{0}^{s_{I}}\left(\hat{u}_{n}+\kappa \hat{u}\right) \mathrm{d} s\right| \sim \delta\left|\int_{0}^{s_{t}} \hat{u} \mathrm{~d} s\right| .
$$

For simplicity, we shall consider below only the extreme cases

$$
\delta \ll \sqrt{C} \ell, \delta \gg \sqrt{C} \ell .
$$

which will be referred to as the small and large surface viscosity limits, respectively, and lead to the additional condition (1.12a) and to

$$
\int_{0}^{s_{L}} \hat{h}(s, t) \mathrm{d} s=0
$$

respectively. For consistency, in the latter case, the effect of the lateral walls should be added as a forcing term on the right-hand side of $(1.5 b)$. Since surface diffusion is neglected, $(1.12 b)$ stands also when modelling annular containers.

The remainder of the paper is organized as follows. Under the above assumptions, the exact formulation (1.1)-(1.6), (1.10) can be simplified significantly, which will be done in $\$ 2$. The simplified equations apply for contamination effects that are not too small as explained in $\$ 3$, where the results for a clean frec surface obtained in MMV are also summarized. The simplified equations will be numerically analysed in $\$ 4$, where the relevant large-time patterns resulting from the primary bifurcations will be obtained; the above-mentioned low and high surface viscosity limits will be considered separately. The paper ends with some remarks on the scope and consequences of the main results.

\section{Coupled spatial phase-streaming flow equations in two dimensions}

We now extend the analysis in MMV to the contaminated case. Thus, only the new ingredients will be emphasized below. $\Lambda$ s in MMV, in order to isolate the effect of the streaming flow in generating drift instabilities (which also can be due to symmetry breaking of wave modulation, see Lapuerta et al. 2002), we consider spatially uniform surface waves. After a transient, these waves exhibit a constant amplitude and become quasi-standing, namely standing in a slowly moving reference frame. Thus, the surface waves are completely determined up to a spatial phase. The aim of this section is to derive a set of equations describing the joint dynamics of the streaming flow and the spatial phase of the surface waves. 
Vibrations produce an oscillatory flow that consists in principle in two counterpropagating surface waves, and is nearly inviscid except in two boundary layers attached to the bottom plate and the free surface. The flow variables outside these boundary layers, the free-surface elevation, and the surfactant concentration are written as

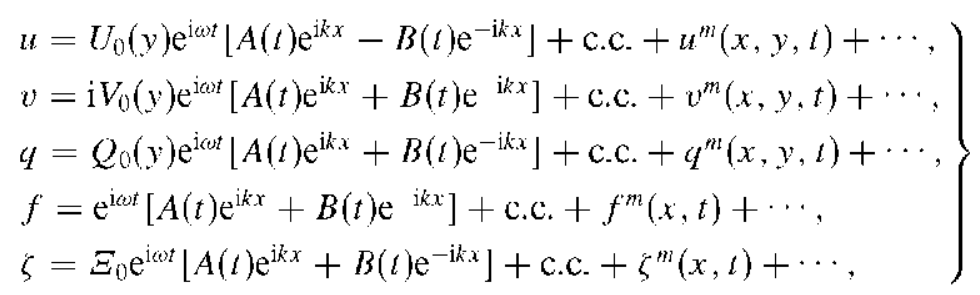

where we have displayed only the leading-order terms associated with the surface waves (assumed to be spatially constant) and the streaming flow, which can be seen as a temporal mean flow and is denoted hereinafter with the superscript $m$; c.c. stands for the complex conjugate and

$$
\begin{aligned}
& U_{0}=-\frac{k Q_{0}}{\omega_{0}}, \quad V_{0}=\frac{Q_{0 y}}{\omega_{0}}, \quad Q_{0}=\frac{\omega_{0}^{2} \cosh k(y+1)}{k \sinh k}, \\
& \omega_{0}^{2}=k\left(1+T k^{2}\right) \tanh k .
\end{aligned}
$$

$\Xi_{0}$ cannot be calculated in the inviscid approximation that leads to (2.2)-(2.3), sce the Appendix. The weakly nonlinear level of our description requires that the complex amplitudes (and the remaining slowly varying quantities) be small and depend weakly on timc, i.c. $\left|\Lambda^{\prime}\right| \ll|\Lambda| \ll 1$ and $\left|B^{\prime}\right| \ll|B| \ll 1$. 'The complex amplitudes $\Lambda$ and $B$ obey the following amplitude equations,

$$
\begin{aligned}
& A^{\prime}=\left[-d_{1}-\mathrm{i} d_{2}+\mathrm{i} \alpha_{3}|A|^{2}-\mathrm{i} \alpha_{4}|B|^{2}-\mathrm{i} \frac{\alpha_{6}}{L} \int_{-1}^{0} \int_{0}^{L} g(y) u^{m} \mathrm{~d} x \mathrm{~d} y\right] A+\mathrm{i} \varepsilon \alpha_{5} \bar{B}, \\
& B^{\prime}=\left[-d_{1}-\mathrm{i} d_{2}+\mathrm{i} \alpha_{3}|B|^{2}-\mathrm{i} \alpha_{4}|A|^{2}+\mathrm{i} \frac{\alpha_{6}}{L} \int_{-1}^{0} \int_{0}^{L} g(y) u^{m} \mathrm{~d} x \mathrm{~d} y\right] B+\mathrm{i} \varepsilon \alpha_{5} \bar{A},
\end{aligned}
$$

where the various coefficients and the function $g$ are given by

$$
\begin{gathered}
d_{1}=\alpha_{1} C^{1 / 2}, \quad d_{2}=\alpha_{2} C^{1 / 2}+\omega_{0}-\omega \\
\alpha_{1}+\mathrm{i} \alpha_{2}=\frac{k \sqrt{i \omega_{0}}}{\sinh 2 k}+\frac{\sqrt{i \omega_{0}}\left(\delta \omega_{0}-\mathrm{i} \gamma\right) k^{3}}{2 \tanh k\left\lfloor\omega_{0} \sqrt{i \omega_{0}}+\left(\delta \omega_{0}-\mathrm{i} \gamma\right) k^{2}\right\rfloor}, \\
\alpha_{3}=2 \omega_{0} k^{2}+\frac{\omega_{0} k^{2}}{4 \sigma^{2}} \frac{\left(9-\sigma^{2}\right)\left(1-\sigma^{2}\right)+\left(7-\sigma^{2}\right)\left(3-\sigma^{2}\right) T k^{2}}{\sigma^{2}+\left(\sigma^{2}-3\right) T k^{2}}-\frac{3 T \omega_{0} k^{4}}{4\left(1+T k^{2}\right)}, \\
\alpha_{4}=\frac{\omega \gamma_{0} k^{2}}{2}\left[\frac{\left(\sigma^{2}+1\right)^{2}}{\sigma^{2}} \frac{1+T k^{2}}{1+4 T k^{2}}+\frac{\left.4+7 T k^{2}\right], \quad \alpha_{5}=\omega_{0} k \sigma}{1+T k^{2}}\right], \quad g(y)=\frac{2 \omega_{0} k \cosh [2 k(y+1)\rfloor}{\sinh ^{2} k}, \\
\alpha_{6}=\frac{k \sigma}{2 \omega_{0}}, \quad
\end{gathered}
$$

and coincide with their counterparts in MMV except for $\alpha_{1}$ and $\alpha_{2}$, which include the eflect of surface contamination, as calculated in the $\Lambda$ ppendix; here, $\sigma=\tanh k$. A plot of the damping ratio, $\alpha_{1}$, in terms of $k$ for the indicated values of $\delta, \gamma$ and $T$ is given in figure 2 . 


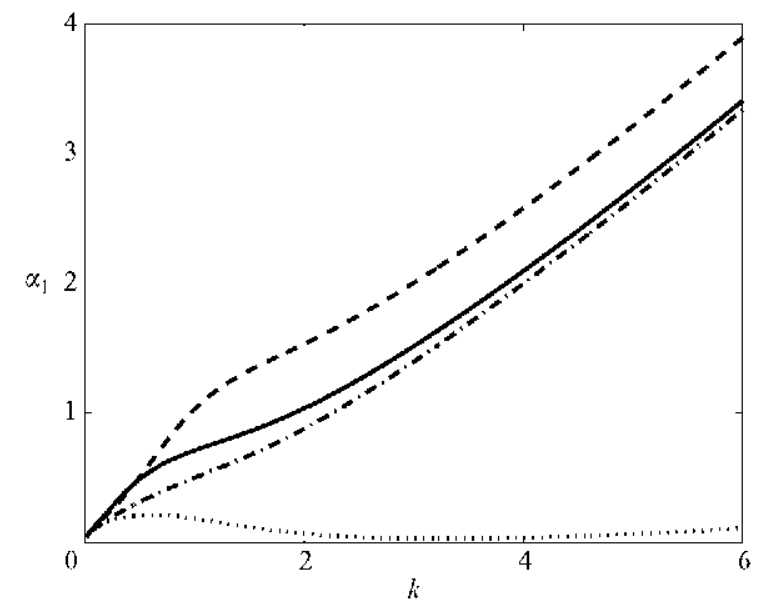
Figure 2. The damping ratio $\alpha_{1}$ in terms of $k$ for $T=7.42 \times 10^{4}$ and,$(\gamma, \delta)=(1,1)$;
,$---(\gamma, \delta)=\left(1,10^{3}\right) ;-\cdot-,(\gamma, \delta)=\left(10^{3}, 1\right) ; \cdots,(\gamma, \delta)=\left(10^{3}, 10^{3}\right)$.

The non-local term in (2.4)-(2.5) accounts for the effect of the streaming flow on the surface wave dynamics. As in MMV, the change of variables

$$
\begin{aligned}
A & =A_{0} \mathrm{e}^{\mathrm{i} k \psi}, \quad B=B_{0} \mathrm{c}^{\mathrm{i} k \psi}, \\
\text { with } \frac{\mathrm{d} \psi}{\mathrm{d} t} & =\frac{\alpha_{6}}{k L} \int_{1}^{0} \int_{0}^{L} g(y) u^{m}(x, y, t) \mathrm{d} x \mathrm{~d} y,
\end{aligned}
$$

eliminates the non-local term from (2.4) (2.5). The resulting system of equations is such that all solutions converge for large time to a steady state of the form

$$
\Lambda_{0}=B_{0}=R_{0} \mathrm{c}^{\mathrm{i} \phi_{0}}
$$

where $\phi_{0}$ is an arbitrary temporal phase and $R_{0}>0$ is given by

$$
R_{0}^{2}=\frac{d_{2} \pm\left(\alpha_{5}^{2} \varepsilon^{2}-d_{1}^{2}\right)^{1 / 2}}{\alpha_{3}-\alpha_{4}}
$$

Ignoring an initial transient, we can substitute (2.11)-(2.13) into (2.1), to obtain

$$
\left.\begin{array}{l}
u=2 \mathrm{i} R_{0} U_{0}(y) \mathrm{e}^{\mathrm{i}\left(\omega t \mid \phi_{0}\right)} \sin k(x-\psi)+\mathrm{cc} .+u^{m}(x, y, t)+\cdots, \\
v=2 \mathrm{i} R_{0} V_{0}(y) \mathrm{e}^{\mathrm{i}\left(\omega t+\phi_{0}\right)} \cos k(x-\psi)+\mathrm{ccc}+v^{m}(x, y, t)+\cdots \\
f=2 R_{0} \mathrm{e}^{\mathrm{i}\left(\omega t \mid \phi_{0}\right)} \cos k(x-\psi)+\mathrm{ccc}+f^{m}(x, t)+\cdots,
\end{array}\right\}
$$

and similar expressions for $q$ and $\zeta$. Thus, the oscillatory part of the flow represents a spatially constant wave that is standing in a slowly moving reference frame, $x=\psi(t)$, where the spatial phase $\psi$ is given by (2.12). As in MMV, this simplifies the streaming flow equations, which become the usual continuity and Navier Stokes equations. The boundary conditions at the lower plate,

$$
u^{m}=-2 \alpha_{7} R_{0}^{2} \sin 2 k(x-\psi), \quad v^{m}=0 \quad \text { at } y=-1,
$$

are again as in MMV, but the upper boundary conditions (which were $u_{y}^{m}=v^{m}=0$ in MMV),

$$
u^{m}=-2 \alpha_{8} R_{0}^{2} \sin 2 k(x-\psi)+u_{0}^{m}(t), \quad v^{m}=0 \quad \text { at } y=0
$$



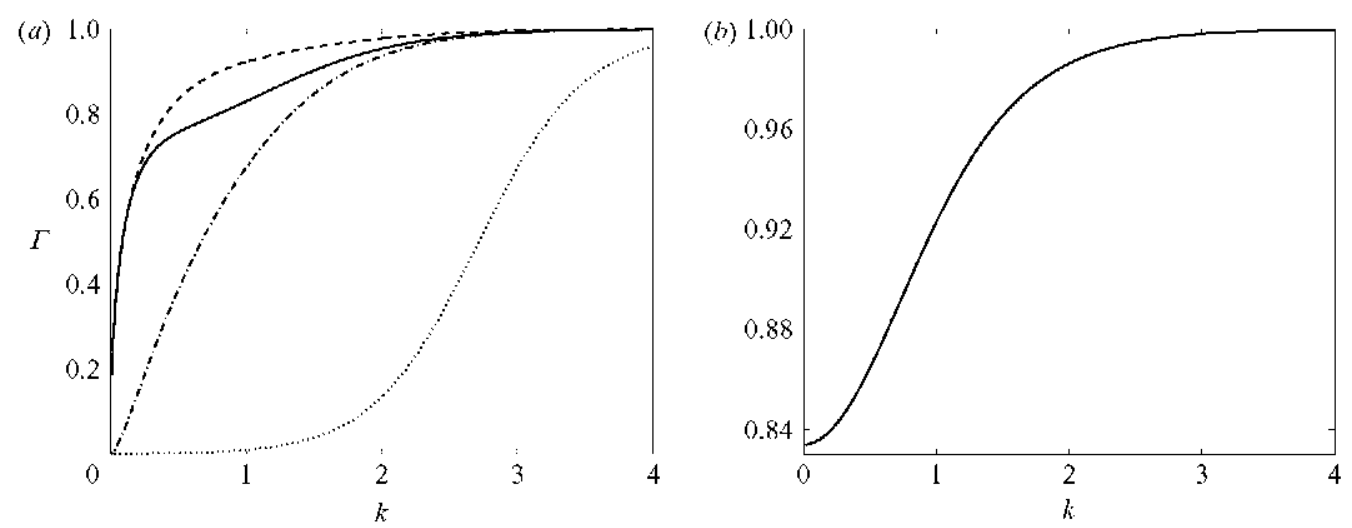

FIGURE 3 . The contamination parameter $\Gamma$, measuring the relative effect of contamination in the generation of the streaming flow. (a) $I$, $v$ s. $k$ for $T=7.42 \times 10^{4}$ and,$-(\gamma, \delta)=(1,1)$; ,$---(\gamma, \delta)=\left(1,10^{3}\right) ;-\cdot-,(\gamma, \delta)=\left(10^{3}, 1\right) ; \cdots ;(\gamma, \delta)=\left(10^{3}, 10^{3}\right)$. (b) The maximum value of $\Gamma \mathrm{ts}, k$ for $T=7.42 \times 10^{4}$ and varying $\gamma$ and $\delta$.

include the effect of surface contamination, which completely changes the structure of the oscillatory boundary layer attached to the free surface, as explained in the $\Lambda$ ppendix. It involves the unknown overall velocity $u_{0}^{m}$, which is determined through one of the following additional conditions resulting from (1.12a) and (1.15): either

$$
\int_{0}^{I .} u_{y}^{m} \mathrm{~d} x=0 \text { at } y=0
$$

in the low surface viscosity casc, as explaincd in the $\Lambda$ ppendix, after $(\Lambda 16)-(\Lambda 17)$, or

$$
u_{0}^{m}=0
$$

in the high surface viscosity casc, as obtaincd by substituting (2.17) into (1.15). The coefficients $\alpha_{7}$ and $\alpha_{8}$ are (see the Appendix)

$$
\begin{gathered}
\alpha_{7}=\frac{3 \omega_{0} k}{\sinh ^{2} k}, \\
\alpha_{8}=\frac{\omega_{0} k}{\tanh ^{2} k}\left(\frac{4\left(\gamma+\mathrm{i} \delta \omega_{0}\right) k^{2}}{\omega_{0} \sqrt{1 \omega_{0}}+\left(\delta \omega_{0}-\mathrm{i} \gamma\right) k^{2}}+\text { c.c. }+\frac{3\left(\gamma^{2}+\delta^{2} \omega_{0}^{2}\right) k^{4}}{\left|\omega_{0} \sqrt{1 \omega_{0}}+\left(\delta \omega_{0}-\mathrm{i} \gamma\right) k^{2}\right|^{2}}\right) .
\end{gathered}
$$

Note that $\alpha_{7}$ and $\alpha_{8}$ are always positive. For convenience, a plot of the parameter

$$
\Gamma=\Gamma(k, T, \gamma, \delta) \equiv \frac{\alpha_{8}}{\alpha_{7}+\alpha_{8}}
$$

in terms of $k$ for the indicated values of $\gamma, \delta$ and $T$ is presented in figure $3(a)$; the selected value of $T$ corresponds to water in a layer of $h=10 \mathrm{~cm}$. $\Gamma$ must not be too small (see \$3) and is a priori bounded between 0 (for $\gamma=\delta=0$ ) and 1 , and, in fact, is quite close to 1 if neither $\gamma$ nor $\delta$ are small and $k$ is moderately large (say $k \geqslant 2$ ). In fact, $\Gamma$ takes values that almost cover the range $0<\Gamma<1$ for realistic values of the parameters, as seen in figure $3(b)$, where the maximum valuc of the function $(\gamma, \delta) \rightarrow \Gamma(k, T, \gamma, \delta)$ is given; note that the maximum value of $\Gamma$ is quite close to 1 except for small $k$. 
Now, for convenience, we introduce the streaming flow Reynolds number

$$
R e=\frac{2 R_{0}^{2}\left(\alpha_{7}+\alpha_{8}\right)}{C},
$$

and rescale time and the streaming flow variables as

$$
\tau=\operatorname{ReC}, \quad \tilde{u}=\frac{u^{m}}{\operatorname{ReC}}, \quad \tilde{v}=\frac{v^{m}}{\operatorname{ReC}}, \quad \tilde{q}=\frac{q^{m}}{(\operatorname{ReC})^{2}},
$$

to rewrite the continuity and Navier Stokes equations, the boundary conditions (2.16) (2.17), and the definition of the spatial phase (2.12), as

$$
\begin{gathered}
\tilde{u}_{x}+\tilde{v}_{y}=0, \\
\frac{\partial \tilde{u}}{\partial \tau}+\tilde{v}\left(\tilde{u}_{y}-\tilde{v}_{x}\right)=-\tilde{q}_{x}+\operatorname{Re}^{1}\left(\tilde{u}_{x x}+\tilde{u}_{y y}\right), \\
\frac{\partial \tilde{v}}{\partial \tau}-\tilde{u}\left(\tilde{u}_{y}-\tilde{v}_{x}\right)=-\tilde{q}_{y}+\operatorname{Re}^{1}\left(\tilde{v}_{x x}+\tilde{v}_{y y}\right), \\
\tilde{u}=-(1-\Gamma) \sin [2 k(x-\psi)], \quad \tilde{v}=0 \quad \text { at } y=-1, \\
\tilde{u}=-\Gamma \sin [2 k(x-\psi)]+\tilde{u}_{0}(\tau), \quad \tilde{v}=0 \quad \text { at } y=0, \\
\tilde{u}, \tilde{v} \text { and } \tilde{q} \quad \text { are } x \text {-periodic, of period } L=2 m \pi / k, \\
\frac{d \psi}{d \tau}=\frac{1}{L} \int_{-1}^{0} \int_{0}^{L} G(y) \tilde{u}(x, y, \tau) \mathrm{d} x \mathrm{~d} y,
\end{gathered}
$$

where the free-surface horizontal velocity is detcrmined from one of the following additional conditions, (2.18) or (2.19), namely either

$$
\int_{0}^{L} \tilde{u}_{y} \mathrm{~d} x=0 \text { at } y=0
$$

for small surface viscosity, or

$$
\tilde{u}_{0}=0 \text {, }
$$

for large surface viscosity. $\Gamma$ is as defined in (2.22), and

$$
G(y)=\frac{2 k \cosh 2 k(y+1)}{\sinh 2 k} .
$$

Equations (2.25)-(2.31) will be callcd coupled spatial phase-streaming flow (CSPSF) equations, and $\mathrm{d} \psi / \mathrm{d} \tau$ can be seen as a drift velocity of the drifting standing waves. Those equations depend on the wavenumber $k$, the spatial period

$$
L=\frac{2 m \pi}{k} \quad \text { with } m=1,2, \ldots,
$$

the contamination parameter $\Gamma$, and the effective Reynolds number $R e$. The latter is proportional to the square of the wave stecpness, $R_{0} k$, which must be small. Since $C$ is also small, $R e$ can vary in a wide range. Assuming that $T$ is not large (which is truc for gravily waves), $\left(R_{0} k\right)^{2} \leqslant 0.1$ and $C \geqslant 10^{6}$, and using (2.3) and (2.34), we obtain the following accessible range for the streaming flow Reynolds number

$$
0 \leqslant R e \leqslant 2 \times 10^{4}\left(\alpha_{7}+\alpha_{8}\right) / k^{2},
$$

which for, e.g. $k=2.37$ gives the range $0 \leqslant R e<2000$ (the same range as in MMV) if both $\gamma$ and $\delta$ are small, and at least the range $0 \leqslant R e<10^{4}$ if either $\gamma$ or $\delta$ are of 
order unity. Note that surface contamination enhances the strength of the streaming flow produced by a surface wave of a fixed (small) stecpness and thus enlarges the range of validity of the approximation. When using the two-dimensional formulation to mimic an annular container, the integration cell corresponds to that part of the container below $m$ wavelengths of the surface wave, which is not necessarily the total length of the container.

\section{Clean free surface $v$ s. small contamination limit}

As mentioned in the previous section, the limit of small contamination ellects in the CSPS $\Gamma$ equations derived above does not coincide with their counterparts considered in MMV. This is because the boundary conditions (2.28) (2.29) reduce to

$$
\begin{aligned}
& \tilde{u}=-\sin [2 k(x-\psi)], \quad \tilde{v}=0 \quad \text { at } y=-1, \\
& \tilde{u}=\tilde{u}_{0}(\tau), \quad \int_{0}^{L} \tilde{u}_{y} \mathrm{~d} x=0, \quad \tilde{v}=0 \quad \text { at } y=0,
\end{aligned}
$$

in the small contamination limit $\Gamma=0$, where for simplicity we have considered the low surface viscosity limil (2.32). These boundary conditions are (3.1) and

$$
\tilde{u}_{y}=0, \quad \tilde{v}=0 \quad \text { at } y=0,
$$

in the clean case considered in $\mathrm{MMV}$, the remaining equations and boundary conditions (2.25) (2.27). (2.30) (2.31) being exactly the same in both limits. This apparent paradox comes from the fact that the validity of the boundary conditions (2.28) (2.29) requires that $\Gamma$ be not too small, namely $\Gamma \gg \sqrt{C}$. For smaller values of $I$, as $I(\sim \gamma+\delta) \sim \sqrt{C}$, a different analysis leads to a homogencous mixed boundary condition at $y=0$ that reduces to (3.2) and (3.3) in the limits $\sqrt{C} \ll \gamma+\delta \ll 1$ and $\gamma+\delta \ll \sqrt{C}$, respectively. 'l'his narrow intermediate regime $(I \sim \sqrt{C})$ is not analysed in this paper because it is not expected to give qualitatively new results, as we illustrate now considering the limiting cases.

(i) For a clean free surface (the limit considered in MMV), the simplified equations are $(2.25)(2.27),(2.30)(2.31),(3.1)$ and (3.3). The bifurcation diagram is given in figure $4(a)$, where the maximum value of the drift velocity $\mathrm{d} \psi / \mathrm{d} \tau$ for the various attractors is plotted $v s$. the Reynolds number for the indicated fixed values of $k$ and $L$. For small values of Re, the basic solution for the streaming flow is stationary, that is, it exhibits no drift ( $\psi=$ constant) and corresponds to a standard standing wave (SW), whose streamlines are qualitatively similar to those in figure $5(a)$, except that the small upper vortices are now absent. $\Lambda t$ Re $=270$, these SWs exhibit a supercritical I Iopf bifurcation that gives limit cycles that correspond to new (drifting) SWs whose nodes oscillate back and forth. This primary bifurcation seems to be present for all $k$ and to remain unchanged at large aspect ratio. The resulting oscillating SWS can be unstable for larger $\operatorname{Re}(\operatorname{Re}>291.5$ in figure 4$)$, where a new branch of oscillating SWs exhibiting different symmetry propertics bifurcates. The new branch in turn becomes unstable for larger $R e(R e=466$ in figure 4$)$, where the system jumps to a new branch of steadily travelling waves (TWs) that exist in the interval $410<R e<620$; see MMV for further details. The latter TWs resemble the steadily rotating structures found experimentally by Douady et al. (1989), where they were quite robust (namely, they were the most frequently non-strictly-standing patterns), while in MMV they were quite sensitive to the various parameters. More complex, chaotic oscillatory patterns are obtained for other values of $k$ and $L$, but are again not robust. 

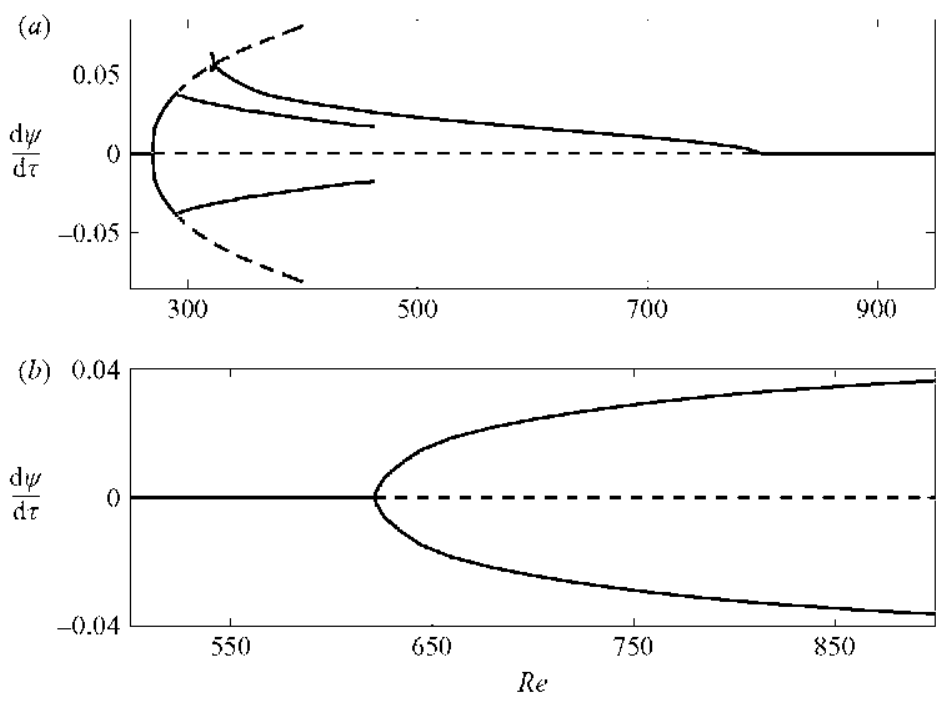

FIGURE 4. Bifurcation diagram of $(a)$ the clean free surface case and $(b)$ the small contamination limit for $k=2.37$ and $L=2.65(m=1)$.
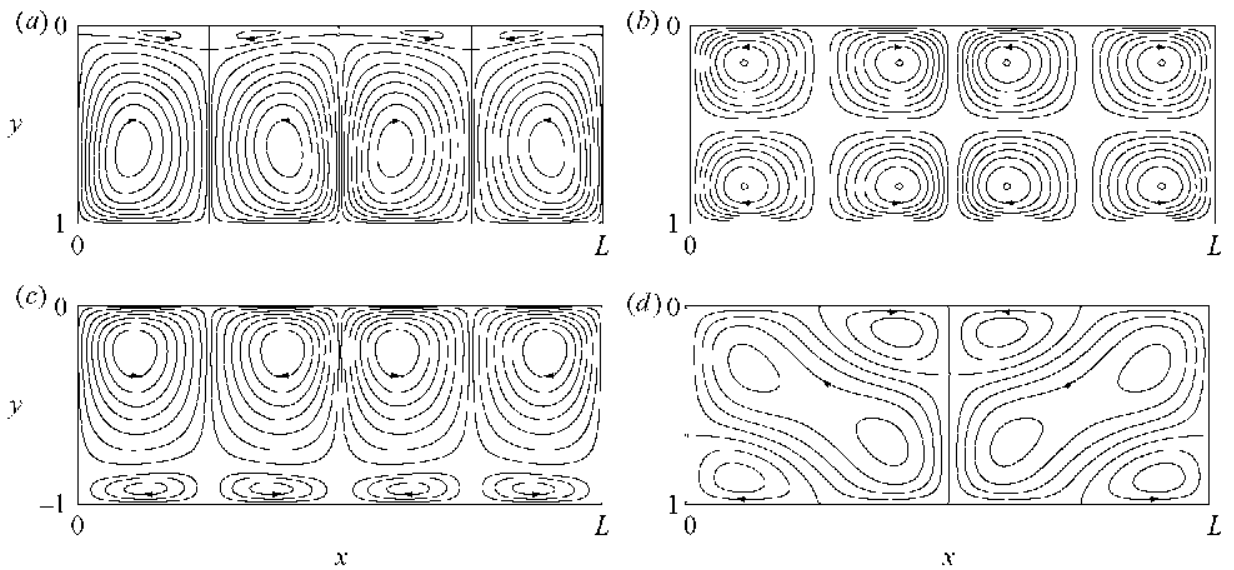

Ficiure 5. Strcamlines of some steady r-symmetric SWs of (2.25) (2.31), for $k=2.37$, $L=2.65(m=1)$, and $(R e, \Gamma):(a)(200,0.1),(b)(160,0.5),(c)(60,0.9)$ and $(d)(200,0.5)$.

(ii) In the small contamination limit case, and the same values of $k$ and $L$, the simplified equations are (2.25) (2.27), (2.30) (2.31) and (3.1) (3.2), and exhibit the bifurcation diagram given in figure $4(b)$, where it is seen that the primary branch of standing waves (with streamlines qualitatively similar to those for the clean case considered above) suffers a Hopf bifurcation at $R e=619.1$, producing limil cycles that remain stable for larger values of $R e$. Note that this diagram is qualitatively similar to that described in figure 7 of $\mathrm{MMV}$, although the wavenumber $k$ and length $L$ are different. Non-symmetric limit cycles and more complex oscillatory patterns are obtained for other values of $k$ and $L$, but these are again not robust.

Comparison of the results described above shows that the basic SWs $(a)$ show qualitatively similar streamlines and (b) are more stable in the low contamination case than in the clean case. This latter conclusion is due to the fact that the no-slip 
boundary conditions (3.2) are more rigid than the free stress boundary conditions (3.3).

\section{Large-time dynamics of the coupled spatial phase-streaming flow equations}

The CSPSF equations (2.25)-(2.31) are invariant under the symmetrics

$$
\begin{gathered}
x \rightarrow x+c, \quad \psi \rightarrow \psi+c, \\
x \rightarrow-x, \quad \tilde{u} \rightarrow-\tilde{u}, \quad \psi \rightarrow-\psi, \quad \tilde{u}_{0} \rightarrow-\tilde{u}_{0}, \\
x \rightarrow x+L / 2 .
\end{gathered}
$$

The first two symmetrics come from the invariance of the original problem (1.1)(1.6) under horizontal translation and reflection. The non-steady reflection-symmetric (r-symmetric) attractors (invariant under (4.2) after a translation), will be referred to as locally or globally r-symmetric depending on whether they are r-symmetric for all $\tau$ or they exhibit an $\mathrm{r}$-symmetric orbit in phase space. Attractors that are invariant under (4.3) will be called $(L / 2)$-symmetric below.

The analysis of the attractors of the CSPS $\Gamma$ equations (2.25)-(2.31) must rely on numerics. The equations have been discretized exactly as in MMV. The dynamics of the CSPSF equations depend on which additional condition, (2.32) or (2.33), is applied. These two conditions become the same for locally r-symmetric solutions, as is the basic steady state that is considered first.

We have four free parameters, $k, m$ (which is an integer and defines the length $L$, see (2.35)), $\Gamma$ and $R e$, which are too many to give a systematic description of the results. Thus, we fix below $k=2.37$ (the value most completely analysed in MMV) and vary $\Gamma$ and $R e$. All results will be obtained first for $m=1$ and then we shall check whether the result depends on $m$.

For small Re, the CSPSF equations become linear and exhibit a unique attractor, which is a $(L / 2)$-symmetric and $\mathbf{r}$-symmetric stcady statc; thus it cxhibits no drift, namely $\psi=$ constant, according to (2.31), and corresponds to a standard SW. The streamlines are as those plotted in figures $5(a)-5(c)$ for $m=1$ and the indicated values of $k, R e$ and $\Gamma$. Note that the flow consists of an array of pairs of counter-rotating vortices. The upper and lower vortices disappear as $\Gamma \rightarrow 0$ and as $\Gamma \rightarrow 1$, respectively. $\Lambda$ lso, the vortices are more and more localized near the boundaries as $k$ increases, namely for increasing values of the forcing frequency; and for large Re, vorticity is localized in two sccondary, lower and upper boundary layers, and in some vertical plumes born near the stagnation points of the forcing velocities. IIowever, these two (obvious) latter statements are not illustrated here to avoid giving too many figures. Since these SWs are locally r-symmetric, they satisfy both conditions, (2.32) and (2.33). For larger $R e$ instead, the dynamics do depend on which additional condition, (2.32) or (2.33), is imposed, and generally involve a complex variety of steady, steadily travelling, periodic, quasi-periodic and chaotic attractors, whose complete description is well beyond the scope of this paper, where the emphasis will be on the primary instabilitics of the basic SWs in figures $5(a)-5(c)$.

In the remainder of the paper, any (steady, periodic, quasi-periodic or chaotic) attractor exhibiting no overall drift, namely, such that the temporal mean value of $\psi^{\prime}$ vanishes, which will frequently happen because such an attractor is globally $\mathbf{r}$ symmetric, will be called a standing wave (SW), while it will be referred to as a travelling wave ('W) if the overall drift is not zcro.

In order to avoid giving too many figures, the steady standing waves will be described below plotting the associated streamlines (figure 5). Similarly, steady 


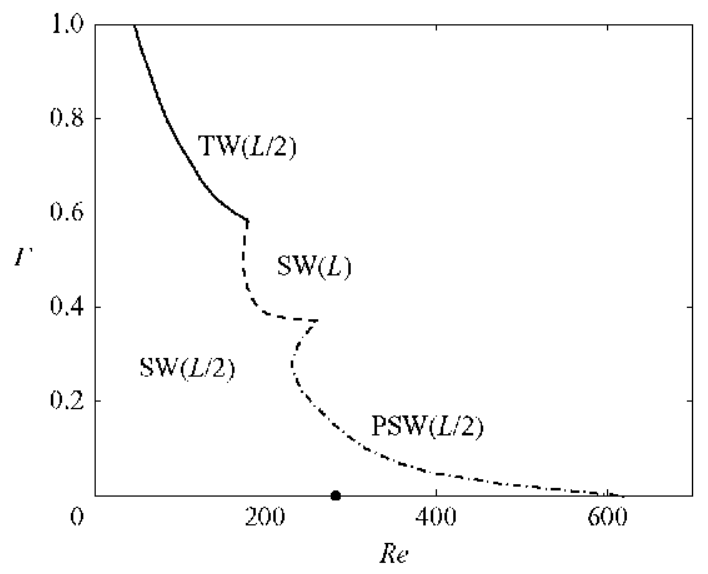

FIgidRe 6. The primary instability of the basie SW of (2.25) (2.31), (2.32), labelled SW( $L / 2)$, for $k=2.37, L=2.65(m=1)$. The bifurcation is either a IIopf bifurcation $(--)$ if $0<\Gamma<0.372$, an $(L / 2)$-symmetry breaking bifurcation $(---)$ if $0.372<\Gamma<0.584$, or a parity breaking bifurcation $(-)$ if $0.584<1<1$.

streamlines in moving axes will be plotted to describe steadily travelling waves (figures 8 and 12), and the constant drift velocity $\psi^{\prime}$ will be indicated in each case. Oscillatory attractors cannot be described with this detail, but we give the plots of both the instantaneous drift velocity $\psi^{\prime} v$ s. time and the instantaneous position of the pattern $\psi$ vs. time (figures 9 and 13); thus the presence of both instantancous and overall drifts will be appreciated. Some of the oscillatory attractors below are chaotic, which is ascertained by calculating the associated Lyapunov exponents.

\subsection{Low surface viscosity}

We consider (2.25)-(2.31), with the additional condition (2.32). The bifurcation diagram, $I$ vs. Re, giving the primary instability of the basic stcady state considered above is given in figure 6 , where can be seen that the critical Reynolds number ranges from 46.5 to 619.1 for $0<\Gamma<1$, and generally decreases as $\Gamma$ increases. The dot in the axis $I=0$ corresponds to the instability limit for the clean free surface (\$3) and indicates a sharp transition from the clean free surface case to the contaminated case, whose precise description would require us to consider the narrow regime (as $\Gamma \sim \sqrt{C} \ll 1$ ) mentioned in $\S 3$. The primary bifurcation from the basic steady state gives the following.

(i) $(L / 2)$-symmctric stcadily travelling waves, labelled ' 'W $(L / 2)$, if $0.584<I<1$, which appear in a standard parity breaking bifurcation (Crawford \& Knobloch 1991). An example of such a TW is plotted in figure $8(a)$. In contrast with the clean case (figure 4a), these TWs appear in a primary bifurcation, and are quite robust. To illustrate the latter statement, we plot in figure 7 the bifurcation diagram for fixed $I '=0.9$ and varying $R e$, where it is seen that the branch of $(L / 2)$-symmetric $T$ 'Ws that appears at $R e=67.7$ shows for increasing $R e$ an $(L / 2)$-symmetry breaking bifurcation, at $R e=568.2$, where a new branch of non- $(L / 2)$-symmetric TWs (two examples are plotted in figure $8 b, c)$ is born. This remains stable except in a short interval $(760<R e<820)$ of more complex dynamics consisting of oscillatory TWs; an example is given in figure $9(a)$, where the non-zero overall drift is clcarly appreciated. 'This bifurcation diagram corresponds to $m=1$, but it remains unchanged for increasing values of $m$ (we have checked the values $m=2,3$, and 10). 


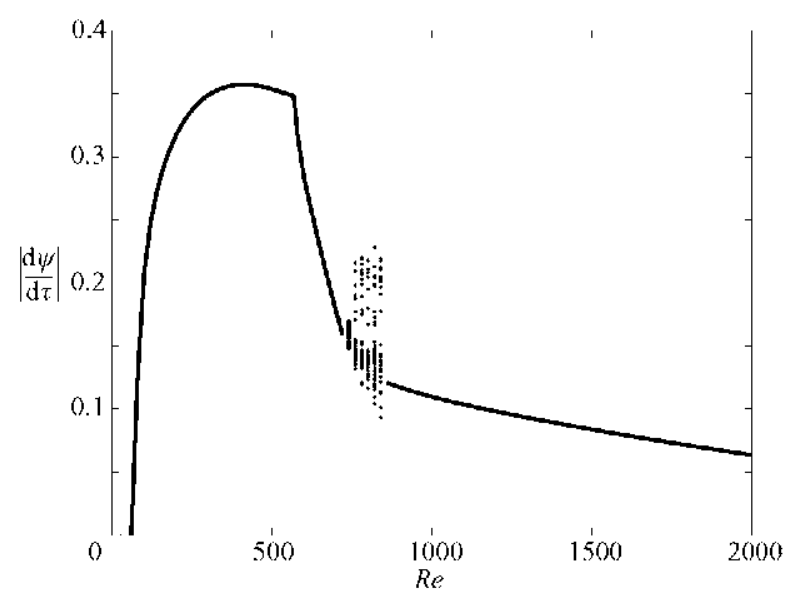

FIgure 7. The bifurcation diagram of the basic SW of (2.25)-(2.31), (2.32) for $k=2.37$, $L=2.65(m=1)$ and $\Gamma=0.9$ lor varying values of $R e$.
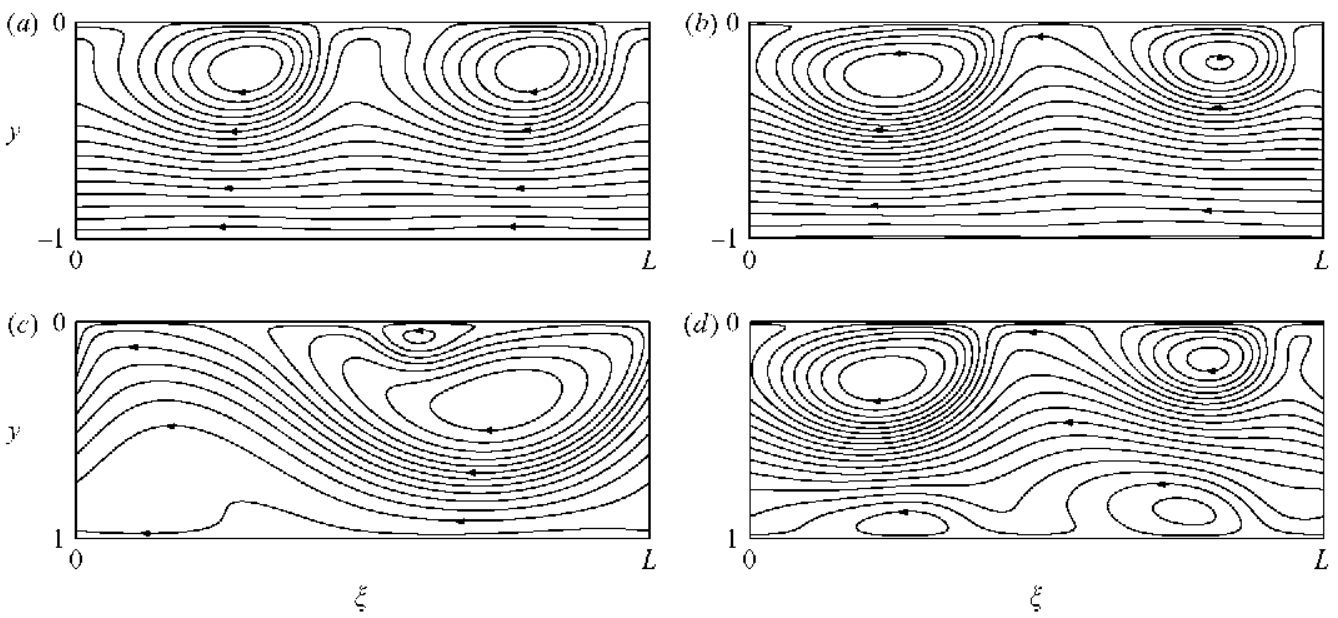

FIGURL 8 . The streamlines for some representative steadily travelling-wave attractors of $(2.25)$ (2.31), (2.32), for $k=2.37, L=2.65(m=1)$ and the following values of $(\operatorname{Re}, \Gamma):(a)(200,0.9)$, (b) $(600,0.9),(c)(860,0.9)$ and $(d)(620,0.65)$. The streamlines are plotted in moving axes $\xi=x-\psi^{\prime} \tau$, with the constant drift velocity $\psi^{i}=0.32,0.27,0.12$ and 0.0015 , respectively (the associated values of $\tilde{u}_{0}$ are $0.49,0.53,0.17$ and 0.15 , respectively).

(ii) r-symmetric but non-( $L / 2)$-symmetric steady standing waves, labelled $\mathrm{SW}(L)$, if $0.372<\Gamma<0.584$, which appear in an $(L / 2)$-symmetry breaking steady bifurcation. $\Lambda$ cxample of such a SW is plotted in figure $5(d)$.

(iii) $(L / 2)$-symmetric periodic standing waves, labelled $\operatorname{PSW}(L / 2)$, if $0<\Gamma<0.372$, which appear in a Hopf bifurcation. Near the threshold, these SWs are globally $r$ symmetric and thus they exhibit no overall drift; an example is plotted in figure $9(b)$. This behaviour is qualitatively similar to that appearing in the non-contaminated case (MMV), sec $\$ 3$.

The bifurcation diagram in figure 6 shows two codimension-two points that deserve some attention: 

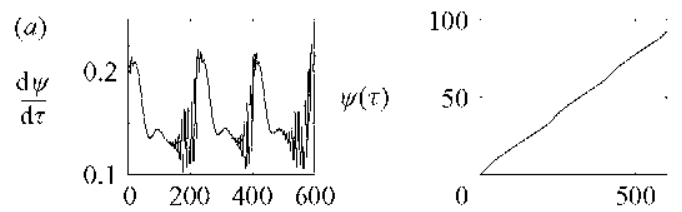

(b)
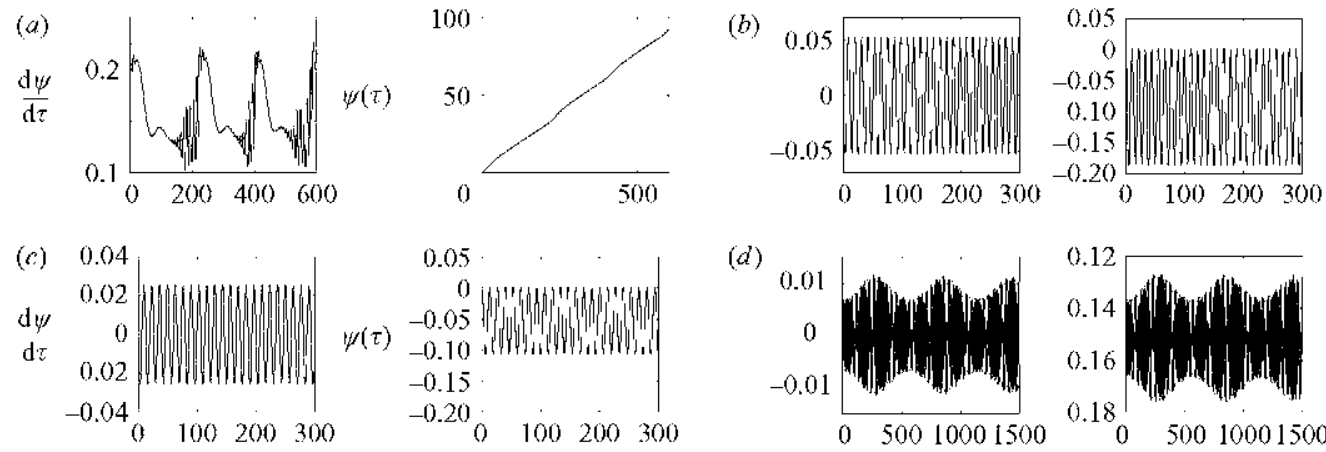

(d)
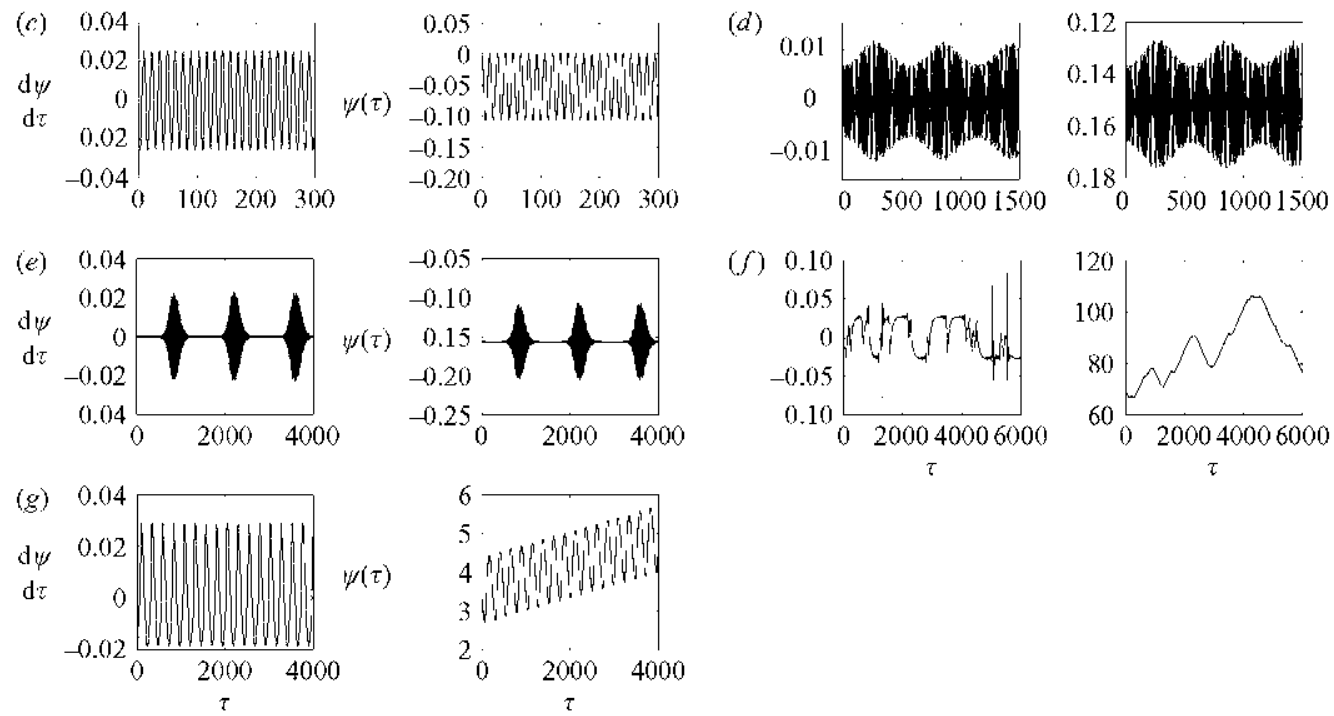

Figure 9. The plots of the drift velocity $\psi^{\prime}$ and the instantaneous position of the pattern $\psi v s$. time for some representative oscillatory attractors of (2.25)-(2.31), (2.32), for $k=2.37, L=2.65$ $(m=1)$ and the following values of $(\operatorname{Re}, \Gamma):(a)(820,0.9),(b)(400,0.1),(c)(270,0.371)$, (d) $(274,0.371),(e)(276.4,0.371),(f)(1440,0.35),(g)(780,0.65)$. Plots $(a)$ and $(g)$ show a non-zero overall drift of the associated pattern (namely, a non-zero overall slope in the plot $\psi r$ vs. $\tau$ ), while the remaining plots show no overall drift.

(i) Near $(R e, \Gamma)=(260.5,0.372)$, the interaction of the steady and periodic SW modes gives a three-dimensional dynamics. The horizontal velocity is written as

$$
\tilde{u}=\tilde{u}^{s}(x-\psi, y, \tau)+\left[X(\tau) \tilde{U}_{0}(x-\psi, y, \tau) \mathrm{c}^{\mathrm{i} s 2 \tau}+\mathrm{c.c} .\right]+b(\tau) \tilde{U}_{1}(x-\psi, y, \tau)+\cdots,
$$

where $\tilde{u}^{s}$ is the basic steady solution, and $\tilde{U}_{0}$ (complex) and $\tilde{U}_{1}$ (real) are the horizontal velocity components of the linear eigenmodes associated with the two interacting modes, and are (L/2)-symmetric and $\mathbf{r}$-symmetric, respectively; $X$ (complex) and $b$ (real) are the small associated amplitudes. Symmetry arguments show that the joint dynamics of $X$ and $b$ are given by

$$
X^{\prime}=\mu_{1} X+\left(\beta_{1}|X|^{2}+\beta_{2} b^{2}\right) X+\cdots, \quad b^{\prime}=\mu_{2} b+\left(\beta_{3}|X|^{2}+\beta_{4} b^{2}\right) b+\cdots,
$$

where $\mu_{1}$ and $\mu_{2}$ are small unfolding parameters that measure departure from the codimension-two point in the plane ( $\left.R e, \Gamma^{\prime}\right)$, and the drift velocity is given by

$$
\psi^{\prime}=\beta_{5} X \mathrm{e}^{\mathrm{i} s 2 \tau}+\text { c.c. }+\cdots .
$$

The calculation of the complex coefficients $\beta_{1}$ and $\beta_{2}$ and the real coefficients $\beta_{3}$ and $\beta_{4}$ is beyond the scope of this paper, but (cf. (2.31)) $\beta_{5}=L{ }^{1} \int_{0}^{L} \int_{-1}^{0} G(y) \tilde{U}_{0} \mathrm{~d} x \mathrm{~d} y$. Note that the dynamics of $a$ and $b$ are decoupled from the phase $\psi$, and that $\psi^{\prime} \neq 0$ (which means that the pattern exhibits a drift) if $X \neq 0$; but the temporal average of the right-hand side of (4.6) vanishes to leading order and thus the overall 
drift is always zero, meaning that all solutions below are SWs. The system (4.5) has been thoroughly analysed clscwhere (Guckenheimer \& Holmes 1983 and references therein). In particular, it is seen that the joint dynamics of $a=|X|$ and $b$ are decoupled to that of the phase of $X$. It follows that

$$
a^{\prime}=\mu_{1} a+\left(\beta_{1} a^{2}+\beta_{2} b^{2}\right) a+\cdots, \quad b^{\prime}=\mu_{2} b+\left(\beta_{3} a^{2}+\beta_{4} b^{2}\right) b+\cdots,
$$

where $\beta_{1}$ and $\beta_{2}$ are real (in fact, the real parts of their complex counterparts in (4.5)), and the phase of $X$, called $\theta$, is such that $\theta^{\prime} \sim a^{2}+b^{2}$ and thus has generally a constant sign, meaning that, e.g. steady states with $a=0$ steady states with $a \neq 0$ and periodic solutions of (4.7) correspond to stcady states, periodic solutions and quasi-periodic solutions of (4.5), respectively. Depending on the values of the coefficients, the system (4.7) can cxhibit the following solutions:

(a) Steady states of the form $\left(a^{s}, 0\right)$ and $\left(0, b^{s}\right)$, which correspond to the periodic and steady SW interacting modes, and are like those already described, and plotted in figures $9(b)$ and $5(d)$.

(b) Steady states of the form $\left(a^{s}, b^{s}\right)$, with $a^{s} \neq 0$ and $b^{s} \neq 0$, which correspond to periodic solutions of (4.5) giving non- $(L / 2)$-symmetric periodic $S W s$ of the system, like that plotted in figure $9(c)$.

(c) Periodic solutions of the form $(a(\tau), b(\tau))$, which correspond to quasi-periodic solutions of (4.5) giving non-( $\mathrm{L} / 2)$-symmetric quasi-periodic SWs of the system, like that plotted in figure $9(d)$; this branch typically disappears at a homoclinic bifurcation illustrated in figure $9(e)$, and consequently no chaotic bchaviour (resulting from further bifurcations on the quasi-periodic branch) is found near this codimension-two point. The system does exhibit chaotic behaviour for $\Gamma$ close to 0.372 , but for much larger values of Re (sec figure $9 f$, where chaoticity is ascertained through Lyapunov exponents).

(ii) Near $(R e, \Gamma)=(179.6,0.584)$ the interaction of the steady standing waves and the steadily travelling waves modes gives a two-dimensional dynamics. The horizontal velocily can bo written as

$$
\tilde{u}=\tilde{u}^{s}(x-\psi, y)+a(t) \tilde{U}_{0}(x-\psi, y)+b(t) \tilde{U}_{1}(x-\psi, y)+\cdots,
$$

where, as above, $\tilde{u}^{s}$ is the basic steady solution, and $\tilde{U}_{0}$ and $\tilde{U}_{1}$ are the horizontal velocity components of the linear marginal eigenmodes associated with the two interacting modes, and arc r-symmetric and $(L / 2)$-symmetric, respectively; $a$ and $b$ are the small, real amplitudes of these modes. Symmetry arguments show that the joint dynamics of $a$ and $b$ are described by the system (4.7) above, where $\mu_{1}$ and $\mu_{2}$ are again small unfolding parameters and the drift velocity is given by

$$
\psi^{\prime}=\beta_{5} b+\cdots .
$$

As above, we do not calculate the coefficients $\beta_{1}, \ldots, \beta_{4}$, but point out that (cf. (2.31)) $\beta_{5}=L^{1} \int_{0}^{L} \int_{-1}^{(0)} G(y) \tilde{U}_{1} \mathrm{~d} x \mathrm{~d} y$. Note that the dynamics of $a$ and $b$ are decoupled from the phase $\psi$, and that $\psi^{\prime} \neq 0$ (which means that the pattern exhibits a drift) if $b \neq 0$. Since the joint dynamics of $a$ and $b$ is as described above, we need only explain the nature of the associated solutions of the system:

(a) The steady states of the form $(a, b)=\left(a^{*}, 0\right)$ and $(a, b)=\left(0, b^{*}\right)$ are now called pure modes and correspond to the two interacting modes. These are qualitatively similar to those plotted in figures $8(a)$ and $5(d)$.

(b) The steady states of the form $(a, b)=\left(a^{s}, b^{s}\right)$ are now called mixed modes and correspond to non-(L/2)-symmetric steadily travelling modes. We have not found 


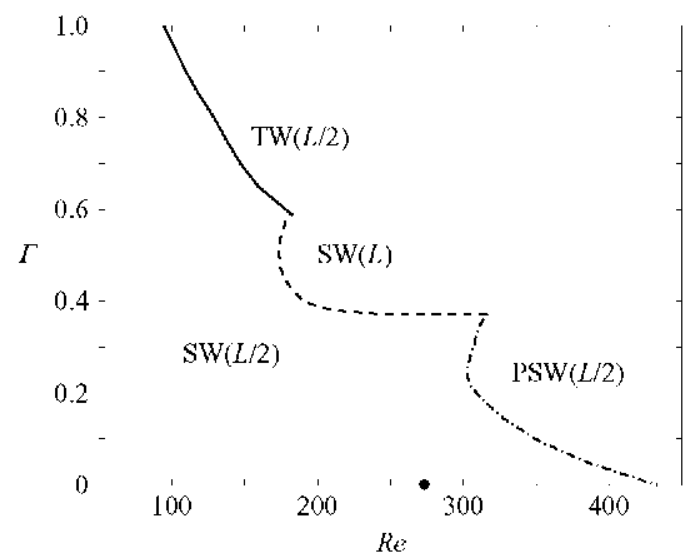

FIGURE 10. The primary instability of the basic SW of (2.25)-(2.31), (2.33), labelled SW(L/2) for $k=2.37, L=2.65(m=1)$. 'The bifurcation is either a IIopf bifurcation $(--)$ if $0<I<0.371$, an $(L / 2)$-symmetry breaking bifurcation $(---)$ if $0.371<I<0.588$, or a parity breaking bifurcation $(-)$ if $0.588<I<1$.

these mixed modes near the codimension-two point, but the system does possess solutions of this type for larger values of $R e$ (see figure $8 d$ ).

(c) The limit cycles of the form $(a, b)=(a(\tau), b(\tau))$ are now periodic solutions and correspond to non-(L/2)-symmetric periodic TWs. Again, we have not found these near the codimension-two point, even though they do exist for larger $R e$ (figure $9 g$ ).

\subsection{IIgh surface viscosity}

Now we consider (2.25) (2.31), with the additional condition (2.33). The counterpart of figure 6 is now plotled in figure 10, where it is secn that the critical Reynolds number ranges from 95 to 440 for $0<\Gamma<1$, and again generally decreases as $\Gamma$ increases. In fact, the intermediate curve plotted with a dashed line is identical to that in figure 7; this is because this instability involves locally r-symmetric SWS, which are such that $\int_{0}^{L} \tilde{u} \mathrm{~d} x=\int_{0}^{L} \tilde{u}_{y} \mathrm{~d} x=0$ and thus satisfy both (2.32) and (2.33); the upper and lower marginal instability curves (plotted with solid and dash-dotted lines, respectively) move to the right and to the left, respectively. Thus, for fixed $\Gamma$, the cffect of surface viscosity is destabilizing at small $I^{\top}$ and stabilizing as $I$ approaches 1 . As in figure 6 , the dot in the axis $\Gamma=0$ corresponds to the instability limit for clean free surface $(\$ 3)$ and, as in $\$ 4.1$ indicates again a sharp transition at small values of $I$.

The primary bifurcation is again as in $\$ 4.1$ and gives:

(i) $(L / 2)$-symmetric steadily travelling waves if $0.588<\Gamma<1$ (an example of such a TW is plotted in figure $12 a$ ). The bifurcation diagram for $\Gamma=0.9$ and varying $R e$ (figure 11) is qualitatively similar to that in figure 6, except that the short interval of complex dynamics is now absent; the $(L / 2)$-symmetry breaking occurs now at $R e=433.2$, and gives non- $(L / 2)$-symmetric steadily travelling waves like that plotted in figure $12 b$. Note that the drift velocity $\psi^{\prime}$ of these TWs (see the caption of figure 12) is much lower than in the low surface viscosity limit. The bifurcation diagram remains unchanged for higher values of $m$ (see (2.35)), which means that these TWs are again quitc robust.

(ii) r-symmetric but non-(L/2)-symmetric steady standing waves, if $0.371<$ $\Gamma<0.588$. The associated streamlines are similar to those plotted in figure $5(d)$. 


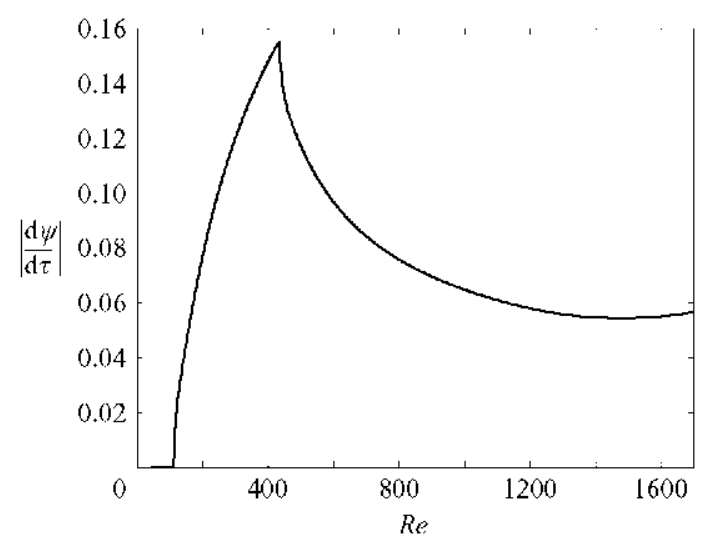

Figure 11. The bifurcation diagram of the basic SW of $(2.25)-(2.31),(2.33)$ for $k=2.37$, $L=2.65(m=1)$, and $I=0.9$ for varying values of $R e$.
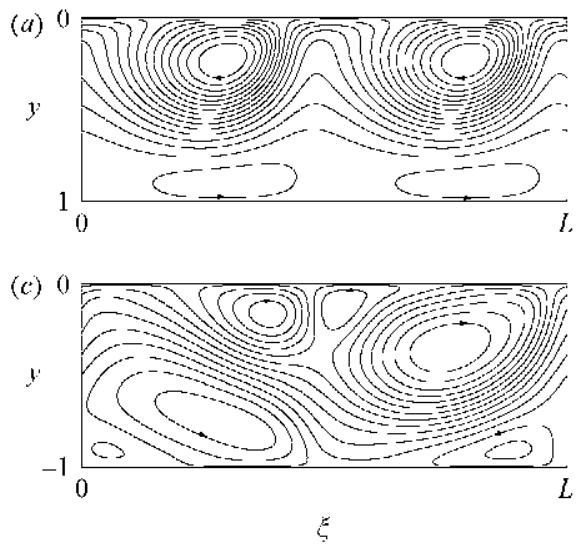

(b)

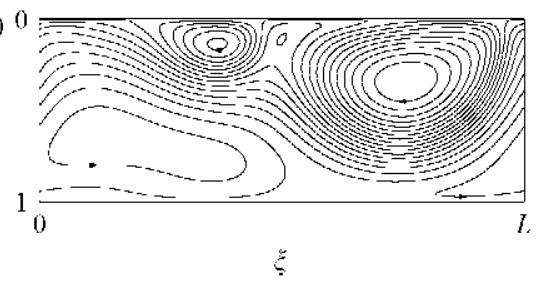

FIGURL 12 . The streamlines for some representative steady and steadily travelling attractors of (2.25) (2.31), (2.33), for $k=2.37, L=2.65(\mathrm{~m}=1)$ and the following values of $(R e, \Gamma)$ : (a) $(200,0.9),(b)(600,0.9)$, and (c) $(500,0.65)$. The streamlines correspond to moving axes $\xi=x-\psi^{\prime} \tau$, with the constant drift velocity $\psi^{\prime}=-0.072,-0.097$ and -0.049 , respectively.

(iii) ( $L / 2)$-symmetric periodic standing waves. The plots of the drift velocity and the instantancous position of the wave are qualitatively similar to those in figure $9(b)$.

The bifurcation diagram in figure 10 shows again two codimension-two points that are similar to those in figure 6 :

(i) Near $(\operatorname{Re}, \Gamma)=(316.2,0.371)$, the interaction of the steady and periodic SW modes is again governed by (4.5) (4.6), and leads to similar dynamics. In particular, a non- $(L / 2)$-symmetric periodic SW gives plots similar to that in figure $9(c)$; and a non-( $L / 2)$-symmetric quasi-periodic SW is as plotted in figure $13(a)$. Ilowever, in contrast with the low surface viscosity case, the branch of quasi-periodic solutions does not disappear near the codimension-two point, but does also exist for much higher values of Re. As we depart from the codimension-two point, this branch of quasi-periodic solutions loses global r-symmetry and cxhibits a non-zero overall drift (an example is plotted in figure 13b) and for still larger $R e$ leads to chaotic dynamics (figure 13d). 

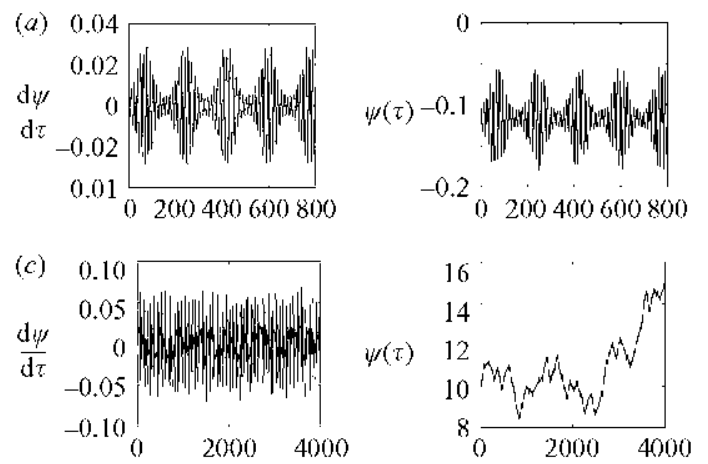

(b)

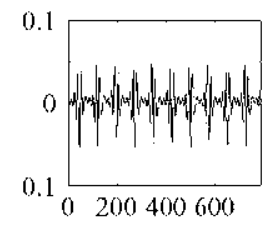

(d)

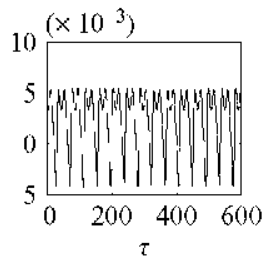

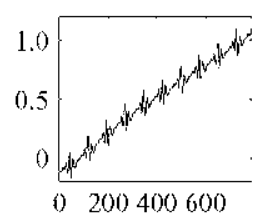

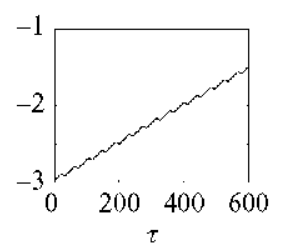

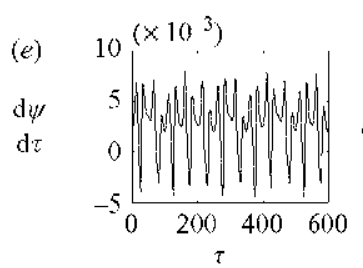

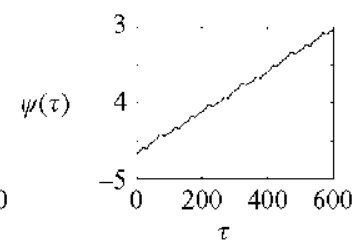

Figure 13. The plots of the drift velocity $\psi^{\prime}$ and the instantaneous position of the pattern $\psi$ $v s$. time for some representative oscillatory attractors of $(2.25)(2.31),(2.32)$, for $k=2.37$, $L=2.65(m=1)$ and the following values of $(R e, \Gamma):(a)(350,0.365),(b)(520,0.365)$, (c) $(760,0.35),(d)(1000,0.65)$ and $(e)(1200,0.65)$. Plots $(b),(d)$ and $(e)$ show a non-zero overall drift of the associated pattern (namely, a non-zero overall slope of the plot $\psi v s . \tau$ ), while the remaining plots show no overall drift.

(ii) Near $(\operatorname{Re}, \Gamma)=(182.5,0.588)$, the dynamics are again given by the amplitude equation (4.7), which yields: (a) pure modes that are qualitatively similar to those plotted in figures $12(a)$ and $5(d)$. $\Lambda$ gain we have found neither mixed modes, nor periodic TWs near the codimension-two point. These do exist for $\Gamma$ close to 0.588 , but require much larger values of $\operatorname{Re}$ (see figures $12 c$ and $13 d$ ); the latter does become chaotic for still larger Re (sec figure 13e).

\section{Conclusions}

We have derived in $\$ 2$ a system of CSPSF equations that give the joint dynamics of the spatial phase of a spatially uniform quasi-standing (namely, standing in a slowly moving reference frame), one-dimensional wave and the two-dimensional streaming flow produced by the wave itsclf. 'This system includes the effect of surface contamination modelled in the simplest possible way. The order of magnitude of the (unknown in contaminated water) Marangoni elasticity has been taken as that already obtained by Nicolás \& Vega (2000) to fit the experimentally measured damping rate for contaminated water. Surface viscosity is expected to be small in contaminated water, but it can also be large in other systems. Thus, we have considered the limiting cases of small and large surface viscosity, see (1.14). I Iowever, in order to compare with the experiment by Douady et al. (1989), we may consider $\delta \ll \gamma \sim 1$, which means that (i) the limit of low surface viscosity is likely to apply (provided that $\sqrt{\mathrm{C}} \ell$ is not too small), and (ii) except for small $k$ gives a value of $\Gamma$ that is close to 1 (see figure $3 b$ ).

We have assumed that $\Gamma \gg \sqrt{C}$, thus ignoring an intermediate narrow regime $\Gamma \sim \sqrt{C}$, discussed in $\$ 3$, in which the transition from the clean free-surface case 
considered in MMV to the limit $\Gamma \rightarrow 0$ of the results in this paper occurs. It is worth noting that surface contamination stabilizes the basic standing waves in this intermediate regime $(\Gamma \sim \sqrt{C})$. This is because the only effect of contamination in this regime on the mean flow is to replace the free stress boundary condition at the frec surface by a no-slip boundary condition, which reduces the strength of the mean flow. The effect of contamination for larger values of $\Gamma \sim 1$ is destabilizing (figures 6 and 10 ). This is because now contamination produces a non-zcro forcing $1 \mathrm{crm}$ for the horizontal velocity of the mean flow, which makes the mean flow stronger as $\Gamma$ increases.

$\Lambda \mathrm{t}$ low surface viscosity, we have found that for increasing values of the contamination parameter $\Gamma$, the basic r-symmetric steady state loses stability either in a Hop bifurcation, a steady symmetry brcaking bifurcation, or in a parity braking bifurcation. The latter bifurcation yields new stable steadily travelling waves that are quite robust, as in the experiment by Douady et al. (1989). The interaction of these three secondary modes yiclds more complex dynamics, which include periodic, quasi-periodic and chaotic attractors, some of them exhibiting a non-zero overall drift. In order to save computational time, we took $m=1$ in (2.35), which means that the computational domain was the fluid under just one wavelength of the surface wave, with periodic boundary conditions in the horizontal direction. The calculated solutions are also solutions for larger $m$, but solutions that are stable for $m=1$ could well be unstable in larger domains due to, e.g. spatial period-doubling instabilities. We have checked that this is not the case, namely that most results stand for larger $m$, as indicated in each checked case. Also, in order to compare with MMV, we took $k=2.37$ in all calculations. This value is lower than the non-dimensional wavenumber in the experiment by Douady et al. (1989), which ranged from 3.3 to 5.1. We did not presented results for these larger values of $k$ because it is not clear that in order to simulate the three-dimensional annular container with our two-dimensional model we should take the same $k$. Instead, the effect of the Stokes boundary layers attached to the lateral walls (which are absent in two dimensions) of the annular domain in generating a mean flow can be somewhat taken into account by decreasing $k$, which increases the relative effect of the Stokes boundary layer attached to the bottom of the container; this eflect is qualitatively similar to that of the lateral walls.

In addition, we have considered the limit of high surface viscosity, to obtain results that are qualitatively similar to those described above; the main difference is that the drift velocity of steadily drifting patterns is much lower at high surface viscosity. Thus we expect that for intermediate values of the surface viscosity, the results will bc also qualitatively similar.

Finally, we point out that the drift of the patterns encountered above occurs in spatially constant surface waves, which are reflection-symmetric to leading order. Thus, the encountered drift is not due to any reflection-symmetry breaking of the surface waves themselves, but to a reflection symmetry breaking of the associated streaming flow. This is in contrast with other sources of drift that are due to a reflection-symmetry breaking of the surface wave envelope, and requires in particular, that the surface waves be not spatially constant (Lapuerta et al. 2002).

We hope that the results in this paper will stimulate further experimental work in the Faraday system in annular containers, with a special attention to the streaming llow, which has becn largely ignored so far.

This work was partially supported by the National Aeronautics and Space Administration (Grant NNCO4GA47G) and the Spanish Ministry of Education (Grant MTM2004-03808). We are indebted to Professor Francisco J. Iiguera for 
illuminating discussions, and to an anonymous referee for suggestions that helped to improve the presentation of the results.

\section{Appendix. Asymptotic derivation of the coefficients $\alpha_{1}, \alpha_{2}$ and $\alpha_{8}$}

The derivation of the coefficients $\alpha_{1}, \alpha_{2}$ and $\alpha_{8}$ requires us to analyse the upper boundary layer attached to the free surface. In fact, $\alpha_{1}$ and $\alpha_{2}$ also include the effect of the lower boundary layer, which is known and will be added a posteriori to the expressions derived below. In the upper boundary layer, attached to the free surface, we use the stretched normal coordinate

$$
n=\frac{n}{\sqrt{c^{c}}}
$$

and introduce the counterparts of the expansions (2.15), namely

$$
\left.\begin{array}{l}
\hat{u}=2 \mathrm{i} R_{0} \hat{U}_{0} \mathrm{e}^{\mathrm{i}\left(\omega t+\phi_{0}\right)} \sin k(x-\psi)+\text { c.c. }+\hat{u}^{m}+\ldots, \\
\left.\hat{v}=2 \mathrm{i}(\omega)+\sqrt{C} \hat{V}_{0}\right) R_{0} \mathrm{c}^{\mathrm{i}\left(\omega t-\phi_{0}\right)} \cos k(x-\psi)+\mathrm{c} . \mathrm{c} .+\hat{v}^{m}+\cdots, \\
q=2\left(1+T k^{2}+\sqrt{C} \hat{Q}_{0}\right) R_{0} \mathrm{e}^{\mathrm{i}\left(\omega t \cdot \phi_{0}\right)} \cos k(x-\psi)+\mathrm{c} . \mathrm{c} .+\hat{q}^{m}+\cdots, \\
f=2 R_{0} \mathrm{e}^{\mathrm{i}\left(\omega t+\phi_{0}\right)} \cos k(x-\psi)+\operatorname{cc} .+\hat{f}^{m}+\cdots, \\
\zeta=2 R_{0} \hat{\Xi}_{0} \mathrm{e}^{\mathrm{i}\left(\omega t+\phi_{0}\right)} \cos k(x-\psi)+\text { c.c. }+\hat{\zeta}^{m}+\cdots,
\end{array}\right\}
$$

where $\hat{u}$ and $\hat{v}$ are defined as in (1.9), and the leading-order constant values of $v$ and $q$ are anticipated. Substituting these and (1.7)-(1.9) into (1.1)-(1.5) and (1.10), we obtain the following system of equations and boundary conditions to determine those terms labelled with the subscript 0 , which are

$$
\begin{gathered}
k \hat{U}_{0}+\hat{V}_{0 \eta}=0, \quad \mathrm{i} \omega_{0} \hat{U}_{0}=-\mathrm{i} k\left(1+T k^{2}\right)+\hat{U}_{0 \eta \eta}, \quad \hat{Q}_{0 \eta}=\omega_{0}^{2} \quad \text { in }-\infty<\eta<0, \\
\hat{V}_{0}=0, \quad \hat{U}_{0 \eta}=-\mathrm{i} \gamma k \hat{\Xi}_{0}-k^{2} \delta \hat{U}_{0}, \quad \hat{Q}_{0}=0, \quad \omega_{0} \hat{\Xi}_{0}+k \hat{U}_{0}=0 \quad \text { at } \eta=0, \\
\hat{U}_{0}=\text { bounded as } \eta \rightarrow-\infty,
\end{gathered}
$$

where we have set $\omega=\omega_{0}$ and for convenience we are anticipating in (A 5) a part of the matching conditions with the outer flow. Integration of $(\Lambda 3)-(\Lambda 5)$ yiclds

$$
\begin{gathered}
\hat{U}_{0}=a\left(1+d \operatorname{cxp}\left(\sqrt{1 \omega_{0}} \eta\right)\right), \quad \hat{V}_{0}=k a\left[\frac{d\left(1-\exp \left(\sqrt{1 \omega_{0}} \eta\right)\right)}{\sqrt{1 \omega_{0}}}-\eta\right], \\
\hat{Q}_{0}=\omega^{2} \eta, \quad \hat{\Xi}_{0}=-\frac{a(1+d) k}{\omega_{0}},
\end{gathered}
$$

where

$$
a=-\frac{\left(1+T k^{2}\right) k}{\omega_{0}}, \quad d=\frac{\omega_{0} \sqrt{\mathrm{i} \omega_{0}}}{\omega_{0} \sqrt{\mathrm{i} \omega_{0}}+\left(\delta \omega_{0}-\mathrm{i} \gamma\right) k^{2}}-1 .
$$

Notc that (cf. (2.2)) $\hat{U}_{0}(\eta=-\infty)=U_{0}(y=0)$ and thus the solution in the boundary layer matches with that in the outer flow.

Now, we derive separately the streaming flow coefficient $\alpha_{8}$ and the linear coeflicients $\alpha_{1}$ and $\alpha_{2}$.

\section{A.1. Derivation of $\alpha_{8}$}

Substituting (1.8)-(1.9) and $(\Lambda 1)-(\Lambda 2)$ into the momentum cquations (1.2)-(1.3), and taking the mean value in the short timescale $t \sim 1$, we obtain, at leading order

$$
\hat{u}_{\eta \eta}^{m}=\hat{q}_{x}^{m}+2 R_{0}^{2}\left(\hat{\hat{U}}_{u \eta} \hat{V}_{0}+\text { c.c. }\right) \sin 2 k(x-\psi),
$$




$$
\hat{q}_{\eta}^{m}=4 R_{0}^{2}\left(\overline{\hat{U}}_{0} \hat{U}_{0 \eta}+\text { c.c. }\right) \sin 2 k(x-\psi) .
$$

Integrating (A9 $b$ ) and substituting into (A9 $a$ ), we obtain

$$
\hat{q}^{m}=4\left|\hat{U}_{0}\right|^{2}+\hat{q}_{0}^{m},
$$

where $\hat{q}_{0}^{m}$ is independent of $\eta$ and is determined by imposing that the right-hand side of $(\Lambda 9 a)$ be bounded as $\eta \rightarrow-\infty$ when taking into account $(\Lambda 10)$. It follows that

$$
\hat{u}_{\eta \eta}^{m}=2 R_{0}^{2}\left[-\hat{U}_{0 \eta} \hat{V}_{0}+\text { c.c. }+2 k\left(\left|\hat{U}_{0}\right|^{2}-\left|\hat{U}_{0}(-\infty)\right|^{2}\right)\right] \sin 2 k(x-\psi) .
$$

Integration of this equation, invoking (A 6 ) and imposing that $\hat{u}^{m}$ be bounded at $\eta=-\infty$ yiclds

where

$$
\hat{u}^{m}(-\infty)=-2 \alpha_{8} R_{0}^{2} \sin 2 k(x-\psi)+u_{0}^{m},
$$

$$
\hat{u}_{0}^{m}=\hat{u}^{m}(\eta=0) .
$$

$u_{0}^{m}$ depends only on $t$ and

$$
\alpha_{8}=\frac{k a^{2}\left[-(4 \mathrm{i} d+\text { c.c. })+3|d|^{2}\right\rfloor}{\omega_{0}},
$$

which invoking $(\Lambda 8)$ lcads to (2.21). Herc, $a$ and $d$ are as defined in $(\Lambda 8)$; the imaginary part of $d$ is positive and thus $\alpha_{8}$ is also positive.

The asymptotic value $(\Lambda 15)$ must be matched with the tangential component of the outer streaming flow velocity at $y=f$, which invoking (1.9) is related with the horizontal component of the streaming flow velocity (again in the outer flow) by

$$
u(y=0)=\hat{u}(y=f)-\left\langle\int_{x} v+\int u_{y}+\ldots\right\rangle,
$$

where $\langle\cdot\rangle$ stands for the temporal mean in the short time scale, $t \sim 1$ and only the leading-order terms are considered. Substitution of $(2.15)$ yiclds $\left\langle f_{x} v+f u_{y}+\cdots\right\rangle=0$ at leading order, and thus the appropriate boundary condition of the outer streaming flow is as given in (2.17).

$\Lambda$ pplying matching conditions with the outer streaming flow velocity, we obtain $u^{n}(y=f) \sim u^{m}(y=0)+\left\langle u_{y}(y=0) f\right\rangle+\ldots$, and taking into account that the temporal mean value of $u_{y}(y=0) f$ is zero at leading order, we obtain the boundary condition (2.17). Also, according to (A 13), since $\left|s_{L}-L\right| / L \ll 1$ and $k L=1(\bmod 2 \pi)$ condition (1.15) is leads to (2.19).

Finally, we show that $(1.12 a)$ leads to (2.18). 'To this end, we first note that the leading-order $\left(O\left(R_{0}^{2}\right)\right)$ part of the stress $u_{y}^{m}$ in the outer flow matches the $O\left(R_{0}^{2} \sqrt{C}\right)$ correction of $\hat{u}_{\eta}^{m}$. Thus, we must also consider this correction, replacing $u^{m}$ with $u_{0}^{m}+\sqrt{C} u_{1}^{m}$ in (A 2a). Replacing this in (1.2) and invoking (1.7) (1.9), we obtain an cquation of the form

$$
\begin{gathered}
u_{1 \eta \eta}^{m}=R_{0}^{2} h(\eta) \sin 2 k(x-\psi) \quad \text { in }-\infty<\eta<0, \\
\hat{u}_{1 \eta}^{m}=R_{0}^{2} h_{0} \sin 2 k(x-\psi) \quad \text { at } \eta=0,
\end{gathered}
$$

where the function $h$ and the constant $h_{0}$ are both $O(1)$ and need not be determined. The form in the right-hand sides of (A 16) and (A 17) follow, just noticing that these terms come from products of $O\left(R_{0}^{2}\right)$ and $O\left(R_{0}^{2} \sqrt{C}\right)$, linear terms that depend on $x$, as do the $O\left(R_{0}\right)$-terms in $(\Lambda 2)$, because both convective terms in (1.2) and nonlincar terms in (1.5b) are reflection-symmetric; thus, terms proportional to $\sin ^{2} k(x-\psi)$ and $\cos ^{2} k(x-\psi)$ are excluded and $u_{\eta}^{m}$ does not contribute to the left-hand side of $(1.12 a)$. 
Integration of (A 16)-(A 17) and applying matching conditions with the solution in the outer llow, taking into account that the spatial mean value (in the short time scale $(t \sim 1)$ of both $u_{y y} f+v_{y} f_{x}$ and $f_{x x} u$ vanish at leading order, readily implies that condition (2.18) holds.

\section{A.2. Derivation of $\alpha_{1}$ and $\alpha_{2}$}

Since these coefficients are associated with a lincar approximation, we need not use a coordinate attached to the boundary in the upper boundary layer. For convenience, we begin with the following linear, viscous equations

$$
\begin{gathered}
k U+V_{y}=0, \\
{\left[\mathrm{i} \omega \omega_{0}-\sqrt{C}\left(\alpha_{1}+\mathrm{i} \alpha_{2}\right)\right] U=-\mathrm{i} k Q+C\left(U_{y y}-k^{2} U\right),} \\
{\left[\mathrm{i} \omega_{0}-\sqrt{C}\left(\alpha_{1}+\mathrm{i} \alpha_{2}\right)\right] V=\mathrm{i} Q_{y}+C\left(V_{y y}-k^{2} V\right),}
\end{gathered}
$$

in $-1<y<0$, with boundary conditions

$$
\left.\begin{array}{c}
U=V=0 \quad \text { at } y=-1, \\
V=\omega_{0}+\mathrm{i} \sqrt{C}\left(\alpha_{1}+\mathrm{i} \alpha_{2}\right), \quad \sqrt{C}\left(U_{y}-k V\right)=-\mathrm{i} \gamma k \Xi-\delta k^{2} U, \\
Q-\left(1+T k^{2}\right)=2 \mathrm{i} C V_{y},\left[\mathrm{i} \omega_{0}-\sqrt{C}\left(\alpha_{1}+\mathrm{i} \alpha_{2}\right)\right] \Xi+\mathrm{i} k U=0 \quad \text { at } y=0,
\end{array}\right\}
$$

which are obtained setting $\varepsilon=0$ in the lincarized version of (1.1)-(1.5) and secking normal modes of the form

$$
\begin{gathered}
u=2 \mathrm{i} R_{0} U / \mathrm{c}^{\mathrm{i}\left(\omega \mid \phi_{0}\right)} \sin k(x-\psi)+\text { c.c. } \\
(v, q, f, \zeta)=2 R_{0}(\mathrm{i} V, Q, 1, \Xi) \mathrm{e}^{\mathrm{i}\left(\omega+\phi_{0}\right)} \cos k(x-\psi)+\text { c.c. }
\end{gathered}
$$

also, we have neglected higher-order terms in the complex damping rate. Note that for small $C$, this problem exhibits two viscous boundary layers attached to the boundaries, and that

$$
U=U_{0}, \quad V=V_{0}, \quad Q=Q_{0},
$$

the inviscid approximations (2.2), outside these boundary layers, while in the upper boundary layer, we have (cf. $(\Lambda 2))$

$$
U=\hat{U}_{0}, \quad V=\mathrm{i} \omega_{0}+\sqrt{C} \hat{V}_{0}, \quad Q=1+T k^{2}+\sqrt{C} \hat{Q}_{0},
$$

where $\hat{U}_{0}, \hat{V}_{0}$ and $\hat{Q}_{0}$ are given by (A 7), in terms of the stretched coordinate (cf. (A 1))

$$
\eta=\frac{y}{\sqrt{c}} \text {. }
$$

The terms $\alpha_{1}$ and $\alpha_{2}$ are readily calculated using the following global solvability condition, first introduced by Nicolás \& Vega (1996) in a related context,

$$
\begin{aligned}
&\left(\alpha_{1}+\mathrm{i} \alpha_{2}\right)\left[\int_{-1}^{0}\left(U_{0} U+V_{0} V\right) \mathrm{d} y+1+T k^{2}\right] \\
&=\sqrt{C} U_{0}(-1) U_{y}(-1)+\frac{\delta \omega_{0}-\mathrm{i} \gamma}{\omega_{0}} k^{2} U_{0}(0) U(0),
\end{aligned}
$$

where $U_{0}$ and $V_{0}$ are the inviscid approximations (2.2) and we have neglected $O(\sqrt{C})$ terms anticipating that $|U| \sim\left|U_{0}\right| \sim|V| \sim\left|V_{0}\right| \sim\left|V_{y}\right| \sim\left|U_{0 y}\right| \sim 1$. This solvability condition is readily obtaince by multiplying $(\Lambda 19)$ and $(\Lambda 20)$ by $U_{0}$ and $V_{0}$, respectively, adding the resulting equations, integrating in $-1<y<0$, integrating by parts and substituting (A 3) and (A 22) (A 22). Now, ignoring at the moment the 
lower boundary layer, and denoting as $\tilde{\alpha}_{1}$ and $\tilde{\alpha}_{2}$ the corresponding values of $\alpha_{1}$ and $\alpha_{2}$, we can use the approximations $(\Lambda 24)$ and $(\Lambda 25)$, to rewrite $(\Lambda 27)$ as

$$
\begin{aligned}
\left(\hat{\alpha}_{1}+\mathrm{i} \hat{\alpha}_{2}\right)\left[\int_{1}^{0}\left(U_{0}^{2}+V_{0}^{2}\right) \mathrm{d} y+1+T k^{2}\right] & =\frac{\delta \omega_{0}-\mathrm{i} \gamma}{\omega_{0}} k^{2} U_{0}(0) \hat{U}(0) \\
& =\frac{k^{2}\left(1+T k^{2}\right)(1+d)\left(\delta \omega_{0}-\mathrm{i} \gamma\right)}{\omega_{0} \tanh k}
\end{aligned}
$$

where $a$ and $d$ are as defined in (A 8), and we have taken into account that the first term on the right-hand side of $(\mathrm{A} 27)$ is $O(\sqrt{C})$ at the upper boundary layer. We need only calculate the integral on the left-hand side, $\int_{1}^{0}\left(U_{0}^{2}+V_{0}^{2}\right) \mathrm{d} y=1+T k^{2}$, to obtain

$$
\hat{\alpha}_{1}+\mathrm{i} \hat{\alpha}_{2}=\frac{k^{3}\left(\delta \omega_{0}-\mathrm{i} \gamma\right)(1+d)}{2 \omega_{0} \tanh k} .
$$

Adding in the right-hand side the well-known (MMV) contribution of the lower boundary layer (which is $k \sqrt{i \omega_{0}} / \sinh 2 k$ ), and replacing (A 8), we obtain the expression of $\alpha_{1}+\mathrm{i} \alpha_{2}$ appearing in (2.7).

\section{REFERENCES}

BoussinESQ, J. 1913 Existence of a superficial viscosity in the thin transition layer separating one liquid from another contiguous fluid. C. R. Hehbd. Seanc. Acad. Sci. 156, 983989.

Crawford, J. D. \& KNobloch, E. 1991 Symmetry and symmetry-breaking bifurcations in fluid dynamics. Anmu. Ren. Fluid Mech. B 23, 341387.

DoRREsten, R. 1951 General linearized theory of the effect of surface films on water ripples. Proc. K. Ned. Akad. Wet. B 54, 260-269.

Douady, S., Fauve, S. \& Thual, O. 1989 Oscillatory phase modulation of parametrically forced surface waves. Europhys. Lett. 10, 309315.

Gockinilimiı, J. \& Hormıs, P. 1983 Nonlinear Oscillations Dynamical Systems and Bifurcation of Vector Fields. Springer.

IJENDERSON, D. M. 1998 Effects of surfactants on laraday-wave dynamics. J. Fluid Mech. 365, 89107.

IJenderson, D. M. \& Miles, J. W. 1994 Surface-wave damping in a circular cylinder with a fixed contact line. J. Fluid Mech. 275, 285299.

IIIglera, M., Vega, J. M. \& KNobloch, E. 2002 Coupled amplitude-mean flow equations for nearly-inviscid Faraday waves in moderate aspect ratio containers. J. Nonlinear Sci. 12, 505551.

IJirsa, A. II., López, J. M. \& Miraghaie, R. 2002 Determination of surface shear viscosity via deep-channel flow with inertia. I. Fluid Mech. 470, 135149.

Jenkins, A. D. \& Dysthe, K. B. 1997 The effective film viscosity of a thin floating fluid layer. J. Fluid Mech. 344, 335337.

Lapuerta, V., MARTel, C. \& VEGA, J. M. 2002 Interaction of nearly-inviscid laraday waves and mean flows in 2-I) containers of quite large aspect ratio. Physica I) 173, 178203.

Levich, V. G. 1962 Physicochemical IIydrodynomics. Prentice-Ilall.

Martín, E., Martel, C. \& Vega, J. M. 2002 Drift inslability of standing Faraday waves. J. Fluid Mech. 467, 57-79.

Miles, J. W. 1967 Surlace-wave damping in closed basins. Proc. R. Soc. Lond. A 297, 459-475.

Nicor Ás, J. A. \& Viri丸, J. M. 1996 Weakly nonlinear oscillations of axisymmetric liquid bridges. J. Fluid Mech. 328, 95-128.

Nicor Ás, J. A. \& Vicis, J. M. $2000 \wedge$ note on the effect of surface contamination in water wave damping. J. Fluid Mech. 410, 367-373.

Scrivin, L. F. 1960 Jynamics of a fluid interface. Chem. kingng Sci. 12, 98108.

VeGA, J. M., KNobloch, E. \& MARTEL, C. 2001 Nearly inviscid liaraday waves in annular containers of moderately large aspect ratio. Physica I) 154, 147171 . 